\title{
Multivariate models from RNA-Seq SNVs yield candidate molecular targets for biomarker discovery: SNV-DA
}

\author{
Matt R. Paul ${ }^{1,5^{*}}$ (D), Nicholas P. Levitt ${ }^{1}$, David E. Moore ${ }^{1}$, Patricia M. Watson², Robert C. Wilson²,3, \\ Chadrick E. Denlinger ${ }^{3,4}$, Dennis K. Watson ${ }^{2,3}$ and Paul E. Anderson ${ }^{1}$
}

\begin{abstract}
Background: It has recently been shown that significant and accurate single nucleotide variants (SNVs) can be reliably called from RNA-Seq data. These may provide another source of features for multivariate predictive modeling of disease phenotype for the prioritization of candidate biomarkers. The continuous nature of SNV allele fraction features allows the concurrent investigation of several genomic phenomena, including allele specific expression, clonal expansion and/or deletion, and copy number variation.

Results: The proposed software pipeline and package, SNV Discriminant Analysis (SNV-DA), was applied on two RNA-Seq datasets with varying sample sizes sequenced at different depths: a dataset containing primary tumors from twenty patients with different disease outcomes in lung adenocarcinoma and a larger dataset of primary tumors representing two major breast cancer subtypes, estrogen receptor positive and triple negative. Predictive models were generated using the machine learning algorithm, sparse projections to latent structures discriminant analysis. Training sets composed of RNA-Seq SNV features limited to genomic regions of origin (e.g. exonic or intronic) and/or RNA-editing sites were shown to produce models with accurate predictive performances, were discriminant towards true label groupings, and were able to produce SNV rankings significantly different from than univariate tests. Furthermore, the utility of the proposed methodology is supported by its comparable performance to traditional models as well as the enrichment of selected SNVs located in genes previously associated with cancer and genes showing allele-specific expression. As proof of concept, we highlight the discovery of a previously unannotated intergenic locus that is associated with epigenetic regulatory marks in cancer and whose significant allele-specific expression is correlated with ER+ status; hereafter named ER+ associated hotspot (ERPAHS).

Conclusion: The use of models from RNA-Seq SNVs to identify and prioritize candidate molecular targets for biomarker discovery is supported by the ability of the proposed method to produce significantly accurate predictive models that are discriminant towards true label groupings. Importantly, the proposed methodology allows investigation of mutations outside of exonic regions and identification of interesting expressed loci not included in traditional gene annotations. An implementation of the proposed methodology is provided that allows the user to specify SNV filtering criteria and cross-validation design during model creation and evaluation.
\end{abstract}

Keywords: Multivariate models, SNV, Biomarker discovery, sPLS-DA, ER+, TNBC, NSCLC, ERPAHS

*Correspondence: mattpaul@mail.med.upenn.edu

1 Department of Computer Science, College of Charleston, 66 George St., Charleston, SC, USA

${ }^{5}$ Department of Cancer Biology, University of Pennsylvania, 421 Curie Blvd,

Philadelphia, PA, USA

Full list of author information is available at the end of the article 


\section{Background}

Defining the molecular basis for complex disease at high resolution has become increasingly important for the discovery of actionable drug targets and the improvement of diagnosis and prognosis of cancer patients. To this end, a widespread approach has been through differential gene expression (DGE) analyses that utilize massively parallel high-throughput RNA sequencing (RNA-Seq). RNA-Seq provides a wealth of information beyond gene expression that can be used to characterize the transcriptome, such as alternative splicing via changes in isoform proportions. Notably, it has recently been shown that single nucleotide variants (SNVs) in the genome can be accurately and reliably called from RNA-Seq data as well [64]. This is significant as previously acquired RNA-Seq data can now be analyzed to determine genotype and provide more biological insight.

It is imperative that SNVs be studied as they molecularly underpin complex disease and phenotype [17]. For example, several SNVs, known to affect major regulatory pathways, have been associated with chemotherapy resistance and survival in lung cancer patients $[39,88]$. In addition to changes in protein structure and function due to mutations in coding sequences of transcripts, SNVs have a variety of functional effects on gene regulation and expression. For example, variants lying in intronic regions can have functional effects on expression by modulating alternative splicing [82].

Furthermore, models created from co-occurring SNV features have also been successful in predicting disease phenotype, such as susceptibility to breast or lung cancer $[51,52]$. These models, however, rely on SNVs that have already been found to be associated with the disease phenotype in question. Herein, we demonstrate that accurate multivariate predictive models which identify and prioritize small subsets of candidate biomarkers can be created from SNV features derived from RNA-Seq data and that a priori knowledge of their phenotypic associations is not necessary for their creation. In fact, because variants with unknown clinical associations are included, novel variants and/or genomic regions can be implicated as candidate biomarkers.

Our proposed methodology seeks to train accurate predictive models on SNV allele fraction (AF) values using sparse projections to latent structures discriminant analysis (sPLS-DA). This approach allows the identification of disease-associated SNVs located in coding regions as well other expressed locations of the genome, such as from intronic, intergenic, and 5' UTR regions, which are often under-represented in cancer biology literature. The continuous nature of SNV AF features from RNA-Seq data also allows the exploration of several genomic phenomena, mainly allele specific expression (ASE), where one allele is preferentially expressed over the other. However, it is important to note that the proposed methodology does not discriminate these events from other sources of allelic imbalance, such as from differential cell survival leading to clonal expansion or depletion and/or from copy number amplification and deletion. In our view, this inherent naïveté is a strength in that these events can be analyzed concurrently in a whole genome fashion, providing a shotgun approach to biomarker discovery. The identification of disease-associated SNVs can thus inform and limit regions of interest when using more comprehensive approaches, such as differential expression, as well as implicate novel unannotated regions that are often ignored using traditional approaches. Furthermore, SNV calling from RNA-Seq avoids using relatively more expensive technology, such as whole-exome and whole-genome sequencing (WES/WGS), and can be used to investigate variations due to RNA-editing, which have been shown to have prognostic value regarding outcomes in cancer [63].

We demonstrate the effectiveness of the proposed methodology and software pipeline, SNV Discriminant Analysis (SNV-DA), on two datasets. The first of which is relatively small dataset of non-small cell lung cancer (NSCLC) primary tumors from which we sought to classify future recurrence. Lung cancer is the leading cause of cancer-related deaths worldwide, with the subtype NSCLC compromising approximately $87 \%$ of lung cancer cases in the United States and causing an estimated 500,000 deaths per year worldwide [26, 32]. Despite advances in diagnosis and clinical treatments, NSCLC continues to be the highest cause of cancer-related deaths in major populations across the world [73]. Thus, it is imperative that a better understanding of the molecular events that drive indolent lung cancers into more aggressive tumors be reached to guide future clinical patient management.

The second dataset included in this analysis is composed of 42 estrogen receptor positive $(E R+)$ and 42 triple negative (TR-) primary breast cancer tumors. Standard targeted therapies for breast cancer rely on the presence of either estrogen, progesterone, and/or Her2/neu receptors in the primary tumor sample [8]. Those tumors that lack these receptors, TR-, are thus resistant to standard approaches and require a combination of chemotherapeutic drugs for their treatment [60]. Compounding this issue, these tumors usually show a more aggressive and metastatic phenotype [28]. Therefore, it is necessary that targets be found that drive triple negative breast cancer to identify effective treatment of this subset of breast cancer.

We show that SNV-DA is able to create multivariate predictive models that accurately predict disease phenotype from variants called from RNA-Seq data and are significantly discriminant towards true sample groupings. We also show that the proposed software pipeline is able to 
identify and prioritize disease-associative SNVs. Importantly, the utility of SNV-DA is supported by the result that rankings produced by the methodology are significantly different than rankings produced from univariate tests and are enriched within genes with significant ASE. Lastly, we present as proof of concept, the discovery of a previously unknown highly mutated ER+ associated hotspot (ERPAHS), which is associated with epigenetic markers in cancer cell lines and whose expression is significantly upregulated in ER+ primary tumors as well as significantly correlated with identified SNV features.

\section{Methods}

First, SNVs are called from processed RNA-seq files using Genome Analysis Toolkit (GATK) [58]. Calls are then filtered by SNPiR tools [64] to remove SNVs that may result from sequencing noise and/or alignment errors. After data transformation, sPLS-DA models are trained on SNVs limited by region of origin. Following the empirical estimation of the optimal number of selected features to be included in the model, performance is evaluating using 10-fold cross-validation. Finally, top predictive SNV features are characterized to determine their relevance to the cancer phenotype in question.

\section{Variant calling pipeline}

The variant calling and filtering pipeline, SNPiR, has been shown to obtain accurate SNVs with minimal false-positives from RNA-Seq data [64]. For each sample, the pipeline consists of several steps: pre- and post-processing, filtering, alignment, and variant calling. Burrows-Wheelers Aligner (BWA) [48] is used with default parameters to map reads as single-end sequences to the human genome (hg19), which is concatenated with exons with known splice junctions as per SNPiR protocol. Samtools and Picardtools are used to remove duplicate and unmapped reads, while GATK [58] is used for indel realignment, base calibration and variant calling using the reference SNP database, dbSNP (NCBI hg19 build 141). SNPiR tools are then used to remove mismatches from the first $6 \mathrm{bp}$ of aligned reads, as well as to remove variant calls from repetitive regions, intronic sites within $4 \mathrm{bp}$ of splice junctions, homopolymer runs, and ambiguously mapped reads determined by BLAT [42].

The resulting output is a BED file containing SNVs with their genomic coordinates and allele fractions. RADAR is first used to determine if SNVs are located at RNAediting sites [67]. The SNV annotation program, ANNOVAR (v2014jul14), is then used to annotate unique SNVs using default parameters [79]. For each SNV, ANNOVAR provides information on the gene and region of origin, which include exonic, intronic, 5' or 3' UTR, intergenic, up/downstream, and non-coding RNA (ncRNA). ANNOVAR defines intergenic variants to those that are at least $2 \mathrm{~kb}$ distal from a coding sequence, whereas the ncRNA category contains variants that do not overlap coding transcript annotations and is used by ANNOVAR to encapsulate both annotated non-coding RNA, such as known miRNA and lncRNA, as well as unannotated loci in the genome. Lastly, Bedtools genomecov [66] is used to determine loci with adequate read coverage using hg19 as reference.

\section{Data transformation and filtering}

The total set of variants is transformed into a matrix $\mathrm{SNVM}$, where $\mathrm{SNVM}_{i, j}$ is the allele fraction of the $i$-th $\mathrm{SNV}$ in sample $j$. Allele fraction, or read-frequency, is defined as the amount of reads supporting the variant allele over the total amount of reads covering that nucleotide position. Read coverages are determined for every $\mathrm{SNVM}_{i, j}$. Those $\mathrm{SNVM}_{i, j}$ values that do not reach the threshold read coverage (default 10) are given a nonavailable (NA) value. Sub-models can then be generated by limiting SNVs to those located in a region of interest, such as exonic positions, and/or by requiring a minimum number of non-zero features.

\section{sPLS-DA and optimal number of features}

Predictive models are created using sPLS-DA, which is implemented in the mixOmics $R$ package $[13,15]$. PLSDA is a supervised, multivariate modeling technique used to determine the variation within $\mathrm{X}$, the SNV data, that is correlated to $\mathrm{Y}$, the class labels (e.g. disease-free versus relapse). The sparse version of the technique, sPLS-DA, seeks to identify the best $K$ features that provides the best discrimination between two classes, ignoring all other features. sPLS-DA thus provides a framework for both feature selection and classification.

Nested cross-validations are used to determine the amount of features, $K$, utilized by sPLS-DA that result in the best predictive performance. For every iteration of 10-fold cross-validation, sub-cross-validations are performed across a range of values for $K$. For each $K$, the model is trained on 10-fold sub-training sets and evaluated. The value of $K$ with the best performance for each iteration of the parent cross-validation is then stored. This process is repeated 15 times to more accurately estimate the distribution of optimal Ks from 150 values. The optimal $K$ is then determined as the rounded value of $K$ that corresponds to the maximum of the estimated kernel density of the distribution of selected $K$ 's, as represented in Fig. 1.

\section{Construction of gene expression models}

To compare the performance of the proposed methodology with traditional gene expression classifiers, models were created using gene expression values as input. For the NSCLC dataset, Bowtie (v1.2.18) [46] and RSEM 


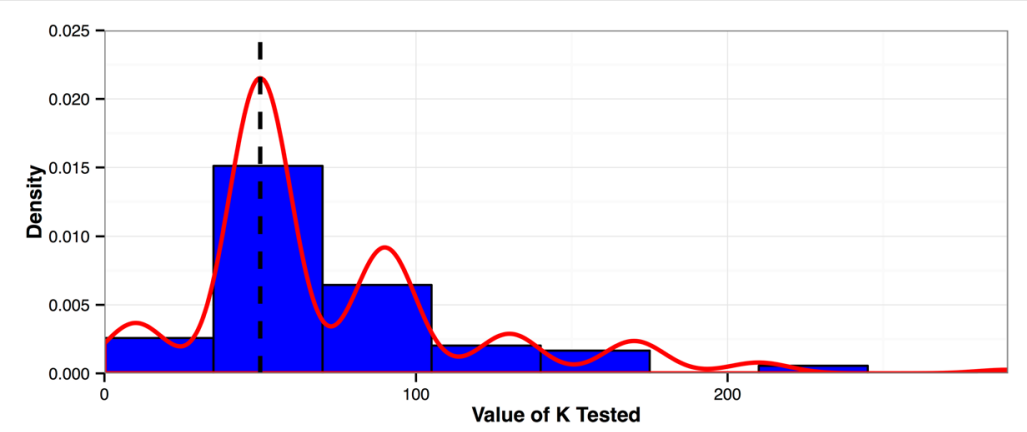

Fig. 1 Selection of Optimal K. A kernel density is estimated from the distribution of $K s$ selected within the nested cross-validations during the creation of each model. The value of $K$ that corresponds to the max of the density is chosen as the optimal value of $K$. The example shown is the distribution of Ks that maximized internal cross validations in the breast cancer exonic SNV model

(v1.2.18) [47] were used with default parameters to align reads to the transcriptome and quantify reads, respectively. For the breast cancer dataset, BWA (v0.7.12) [48] and featureCounts (v1.4.6) [49] was used with default parameters to align reads to the genome and quantify reads, respectively. For both datasets, read counts were normalized via DESeq2 (v1.10.0) [54]. Herein, adjusted $p$-values reported by DESeq2 will simply be referred to as $p$-values. Models were trained on subsequent gene expression matrices using the same parameters as those used in the creation of SNV models. For each dataset, the distribution of performance statistics are compared to that of the corresponding SNV model to identify the similarity of performance between the proposed methodology and the traditional approach.

\section{Evaluation}

After the empirical estimation of the optimal value of $K$, the model is then evaluated using fifteen 10 -fold cross-validations to determine performance via its predictive accuracy, classification sensitivities, and area under the receiver operating characteristic curve (AUC), which seeks to quantify the relationship between true and false positive rates. Though sPLS-DA is able to train a model on features that include NA values, missing data in the test set is not compatible with the resulting model. Therefore, NA values are replaced with the mean of the means of the centered and standardized AF values for each feature within each group in the training set. For example, the mean of the normalized AF values for feature $X$ in group $A$ is averaged together with the mean of normalized AF values for feature $X$ in group $B$ disregarding samples from the test set. This value is then used as a proxy for the missing data in the test set.

To determine if the proposed methodology is discriminant towards the true grouping of disease phenotype, permutation tests are repeated 1000 times to construct the null distribution of model performance (i.e., no relation to phenotype) for each model. The true model performance is then compared to this null distribution to determine significance, with a significantly discriminant model outperforming the majority of permutation test models. Otherwise, it could be said that model performance is independent of the true grouping and is, thus, insignificant. For each test, one iteration of a 10-fold crossvalidation is used to train and test models with randomly permuted sample group labels using the optimal $\mathrm{K}$ that was used in the true model. The number of models with AUC greater than or equal to the true model AUC is divided by the number of tests to determine permutation test $p$-values.

Lastly, to obtain the final set of putative SNV features, the model is trained using all samples and the optimal value of $K$. The selected features are then ranked by the absolute values of their predictive coefficients (or loadings) as determined by sPLS-DA. In order to assay the utility of the proposed methodology, a Friedman rank sum test is used to compare the rankings of selected features to those of traditional approaches - the univariate nonparametric tests, Fisher's exact and Wilcoxon rank sum.

The Fisher's exact test is implemented by the production of a $2 \times 4$ table for each SNV locus, where each value corresponds to the number of samples in each group with detectable levels of each allele in $(A, C, G, T)$, while disregarding samples with sub-threshold read coverage $(<10)$ at that locus. As the presence of an allele is binary in this case, the test only takes into account the differential abundance of the alleles across groups. Whereas, Wilcoxon rank sum test p-values are produced by comparing the distributions of continuous allele fractions and do not directly include information on their differential abundance across samples.

To determine if the proposed methodology selects SNVs that lie in genes that have significant allele-specific expression, selected SNVs were analyzed using MBASED: a method that combines evidence across multiple SNVs 
to identify gene-level ASE [56]. Though the method was designed for the integration of expression data with exonic SNV calls from WES and/or WGS, we applied the methodology on SNVs selected during the creation of our SNV genic models: exonic, intronic, and 3'UTR. To determine if genes from which selected SNVs are located are enriched for ASE, we compared the number of significant ASE gene/sample pairs to those found in equally sized random subsets of genes from which the total set of SNVs were called. One thousand subsets were evaluated to determine the null distribution from which enrichment $p$-values can be computed.

Finally, the top 15 features selected by SNV-DA are characterized by their relevance to cancer phenotype and are analyzed via hierarchical clustering to visualize the co-occurrence of features.

\section{Case studies}

\section{Disease outcome in non-small cell lung cancer}

NSCLC is the leading cause of cancer-related mortality in the US. Adenocarcinoma, the most frequent histological subtype, accounts for $40 \%$ of such deaths [74]. RNA samples were collected from 21 different lung adenocarcinoma tumors with known clinical outcomes obtained from the American College of Surgery Oncology Group (ACOSOG). Since the RNA specimens were received from ACOSOG with no personal identifying information, the local IRB has considered the proposed project "not human subject research" after reviewing the protocol (IRB Pro00013739). Ten of the RNA samples were derived from patients who developed cancer recurrence within three years of their initial surgical resection (Relapse; R). The remaining eleven patients had remained disease free (DF) after three years. Using these samples, we sought to determine the ability of the proposed methodology to identify and prioritize candidate biomarkers that may help predict relapse phenotype in NSCLC.

RNA integrity was verified on an Agilent 2200 Bioanalyzer (Agilent Technologies, Palo Alto, CA). One hundred to two hundred ng of total RNA was used to prepare RNA-Seq libraries using the TruSeq RNA Sample Prep Kit following the protocol as described by the manufacturer (Illumina, San Diego, CA). Three samples per lane were clustered on a cBot as described by the manufacturer (Illumina, San Diego, CA). Clustered RNA-Seq libraries were paired-end sequenced with $2 \times 100$ cycles on a HiScanSQ. Demultiplexing was performed utilizing CASAVA to generate the Fastq files. Each sample produced approximately 25 million reads after sequencing. One sample from the relapse group was removed from subsequent analysis after being identified in our previous study as an outlier based on principle component analyses of expression and alternative splicing [2]. The removal of this sample is additionally supported by the iLOO outlier detection algorithm [27]. Using normalized counts from DESeq2 [54] of all relapse samples, the algorithm identified 567 outlying gene features in the suspect sample - 5.74 standard deviations greater than the distribution of the number of outlying features in the other samples $($ mean $=143.44$, standard deviation $=73.82)$.

\section{Hormone receptor status in breast cancer}

To further validate our model, we obtained a dataset from the publicly available SRA database (SRP042620), which was provided by Varley et al., 2014 [78]. In their publication, the authors sought to identify read-through transcripts that are significantly correlated with breast cancer and/or hormone receptor status. RNA-Seq was obtained from $42 \mathrm{ER}+$ and $42 \mathrm{TR}$ - primary tumors using poly-A capture and Tn-RNA-Seq for library construction. Libraries were sequenced on the Illumina HiSeq 2000 using 50 bp paired-end reads, which produced 50 million reads on average. Instead of trying to predict some future outcome of the patients from which these tumors were sampled, we sought to identify SNV features that co-occur with hormone receptor status. Selected SNVs may thus provide insight into molecular mechanisms differentiating these two subgroups of breast cancer.

\section{Results and discussion Called SNVs}

After variant calling and SNPiR post-processing, 96,025 and 213,020 unique variants with read coverages $>=10$ were found in the NSCLC and breast cancer datasets, respectively. SNV matrices were created by limiting SNVs to those that had at least 3 non-zero values across samples in the NSCLC dataset and 6 non-zero values in the breast cancer dataset. Tables 1 and 2 show the distributions of SNVs for each dataset based on region of origin as determined by RefSeq annotations.

Table 1 Lung: Distribution of SNVs by Region

\begin{tabular}{lll}
\hline Region & Total set & Filtered set \\
\hline 3' UTR $^{\prime}$ & 27,626 & 12,570 \\
exonic & 25,788 & 10,870 \\
intronic & 21,706 & 4,847 \\
nonsynonymous exonic & 11,804 & 4,334 \\
intergenic & 12,867 & 2,460 \\
ncRNA & 4,062 & 1,285 \\
5' UTR $^{\prime}$ & 2,519 & 928 \\
up/downstream & 1,669 & 448 \\
RNA-editing & 1,627 & 430 \\
All SNVs & 96,025 & 33,467 \\
\hline
\end{tabular}

The distribution of called SNVS by region of origin in the NSCLC dataset. A filtered set is created by only including SNVs that have at least three samples with non-zero allele fractions 
Table 2 Breast: Distribution of SNVs by Region

\begin{tabular}{lll}
\hline Region & Total set & Filtered set \\
\hline intronic & 93,860 & 12,378 \\
3' UTR & 33,800 & 9,034 \\
exonic & 31,235 & 6,195 \\
nonsynonymous exonic & 12,598 & 2,484 \\
intergenic & 26,764 & 2,341 \\
ncRNA & 10,767 & 1,767 \\
up/downstream & 3,189 & 491 \\
5' UTR & 2,148 & 419 \\
RNA-editing & 1,902 & 215 \\
All SNVs & 213,020 & 31,788 \\
\hline
\end{tabular}

The distribution of called SNVs by region of origin in the breast cancer dataset. A filtered set is created by only including SNVs that have at least six samples with non-zero allele fractions

\section{Model performance}

sPLS-DA was used to create models using different subsets of SNVs based on type (as shown in Tables 1 and 2) over different ranges of $K$. For the NSCLC dataset, the classification target was patient relapse within a 3 year period, labeled as Relapse, R, or Disease Free, DF. For the breast cancer dataset, the model sought to classify each sample as being from either the cancer subtype estrogen receptor positive, $\mathrm{ER}+$, or hormone receptor triple negative, TR-.

\section{Disease outcome in non-small cell lung cancer}

Table 3 contains measures of performance for models trained on different subsets of SNVs in the NSCLC dataset. What is immediately apparent is that the nonsynonymous exonic model had the best performance by a large margin. The model performed better than chance as seen by its AUC and predictive accuracy. Furthermore, permutation tests reveal that the performance of the model is dependent on the true label groupings $(p=0.016)$, thereby, suggesting that selected SNVs reflect a true biological phenomenon. With the addition of synonymous variants in the model, however, performance reflects that of a failed model. Interestingly, the distribution of AUCs from the nonsynonymous exonic model was significantly better than the distribution of AUCs from the gene expression model (Student's t-test, $p<0.001$ ). Though some of the other models have AUC values that seem to be better than chance, their performances are not significant based on permutation test values.

\section{Hormone receptor status in breast cancer}

Table 4 contains measures of performance for models trained on different subsets of SNVs in the larger breast cancer dataset. Strikingly, all models tested have high AUC distributions and high predictive accuracies. In contrast to the NSCLC dataset, the addition of synonymous SNVs in the exonic model produced significantly better performance (Student's t-test, $p<0.001$ ), while selecting roughly half as many features. The distributions of AUC values from the intergenic and all-SNVs models were significantly higher than from all other models (Student's t-test, $p$ s $<0.001)$ - being able to accurately predict TR-samples 96 and $97 \%$ of the time. Interestingly, models with relatively small amounts of starting features (5' UTR, up/downstream models, and RNA-editing) were also able to produce accurate results. Most importantly, the biological significance of these models is supported by the result that all had permutation test $p$-values $<0.001$. Of note, the model trained on gene expression features had

Table 3 NSCLC model performances

\begin{tabular}{|c|c|c|c|c|c|c|c|}
\hline Model & $\begin{array}{l}\text { Tested Range of K, Every } \\
\text { Nth }\end{array}$ & Opt. K & $\mathrm{A} \cup \mathrm{C}[95 \% \mathrm{Cl}]$ & $P$-value & Pred. Accuracy & DF Sens. & R Sens. \\
\hline $\begin{array}{l}\text { nonsynonymous } \\
\text { exonic }\end{array}$ & $10-1000,40$ & 841 & 0.874 [0.845-0.903] & 0.016 & 0.803 & 0.836 & 0.763 \\
\hline $3^{\prime}$ UTR & $10-1000,40$ & 46 & $0.720[0.650-0.789]$ & $>0.10$ & 0.626 & 0.626 & 0.626 \\
\hline up/downstream & $10-400,15$ & 138 & $0.708[0.690-0.725]$ & $>0.10$ & 0.653 & 0.690 & 0.615 \\
\hline all-SNVs & $10-1000,40$ & 139 & $0.643[0.615-0.671]$ & $>0.10$ & 0.587 & 0.679 & 0.474 \\
\hline intronic & $10-1000,40$ & 59 & 0.634 [0.599-0.669] & $>0.10$ & 0.607 & 0.558 & 0.667 \\
\hline RNA-editing & $10-400,10$ & 36 & $0.615[0.577-0.653]$ & $>0.10$ & 0.573 & 0.503 & 0.659 \\
\hline exonic & $10-1000,40$ & 54 & $0.580[0.552-0.609]$ & $>0.10$ & 0.450 & 0.612 & 0.252 \\
\hline intergenic & $10-1000,40$ & 60 & $0.561[0.519-0.602]$ & $>0.10$ & 0.487 & 0.642 & 0.296 \\
\hline ncRNA & $10-1000,40$ & 36 & $0.556[0.520-0.591]$ & $>0.10$ & 0.547 & 0.721 & 0.333 \\
\hline 5' UTR & $10-750,30$ & 16 & $0.242[0.215-0.268]$ & $>0.10$ & 0.290 & 0.394 & 0.163 \\
\hline gene expression & $10-1000,40$ & 592 & 0.824 [0.803-0.845] & 0.068 & 0.740 & 0.764 & 0.711 \\
\hline
\end{tabular}

The range of $\mathrm{K}$ tested, the optimal value of $\mathrm{K}, \mathrm{AUC}$ and $95 \%$ confidence interval, $p$ value from 1000 iteration permutation tests, predictive accuracy, and classification sensitivities of the top-performing models by genomic region 
Table 4 Breast model performances

\begin{tabular}{|c|c|c|c|c|c|c|c|}
\hline Model & $\begin{array}{l}\text { Tested Range of K, Every } \\
\text { Nth }\end{array}$ & Opt. K & $\mathrm{AUC}[95 \% \mathrm{Cl}]$ & $P$-value & Pred. Accuracy & ER+ Sens. & TR-Sens. \\
\hline intergenic & $10-1000,40$ & 771 & $0.975[0.972-0.977]$ & $<0.001$ & 0.939 & 0.922 & 0.956 \\
\hline all-SNVs & $10-1000,40$ & 163 & 0.972 [0.969-0.975] & $<0.001$ & 0.941 & 0.936 & 0.968 \\
\hline up/downstream & $10-400,15$ & 386 & 0.960 [0.959-0.962] & $<0.001$ & 0.915 & 0.910 & 0.920 \\
\hline exonic & $10-1000,40$ & 50 & 0.958 [0.952-0.964] & $<0.001$ & 0.912 & 0.906 & 0.917 \\
\hline 3' UTR & $10-1000,40$ & 129 & 0.939 [0.936-0.942] & $<0.001$ & 0.884 & 0.914 & 0.854 \\
\hline ncRNA & $10-1000,40$ & 911 & 0.939 [0.936-0.942] & $<0.001$ & 0.843 & 0.917 & 0.768 \\
\hline 5' UTR & $10-400,15$ & 370 & 0.939 [0.931-0.946] & $<0.001$ & 0.837 & 0.873 & 0.801 \\
\hline intronic & $10-1000,40$ & 315 & 0.935 [0.933-0.937] & $<0.001$ & 0.879 & 0.829 & 0.930 \\
\hline $\begin{array}{l}\text { nonsynonymous } \\
\text { exonic }\end{array}$ & $10-1000,40$ & 92 & 0.920 [0.915-0.926] & $<0.001$ & 0.869 & 0.857 & 0.881 \\
\hline RNA-editing & $10-200,5$ & 12 & 0.878 [0.873-0.883] & $<0.001$ & 0.820 & 0.747 & 0.894 \\
\hline gene expression & $10-1000,40$ & 472 & 0.985 [0.983-0.987] & $<0.001$ & 0.963 & 0.976 & 0.951 \\
\hline
\end{tabular}

The range of $\mathrm{K}$ tested, the optimal value of $\mathrm{K}, \mathrm{AUC}$ and $95 \%$ confidence interval, $p$ value from 1000 iteration permutation tests, predictive accuracy, and classification sensitivities of the top-performing models by genomic region

significantly better performance than all models trained on SNV features (Student's t-test, $p<0.001$ ), however, the all-SNVs model surpassed the gene expression model when classifying TR- samples (Student's t-test, $p=0.018$ ), which in this dataset can be considered the experimental group. Furthermore, only one of the top 15 features selected by the gene expression model corresponds to a gene where from a top predictive SNV feature is found: ZNF552.

\section{Predictive SNV features: disease outcome in non-small cell lung cancer}

The rankings of selected nonsynonymous features in the NSCLC dataset were significantly different than univariate rankings from Fisher's exact and Wilcoxon rank sum tests as determined by Friedman rank sum tests $\left(p s<10^{-16}\right)$. Figure 2 visualizes allele fraction distributions of the top 15 predictive SNVs identified during the creation of nonsynonymous exonic SNV model. SNVs chosen are more abundant in one of the groups and/or have higher AF values. Both classes were equally representative in the top selected SNV features (7 DF vs 8 R). Figure 3 contains a heatmap produced during hierarchical clustering analysis of the top 15 selected features. Not surprisingly, SNV-DA was able to prioritize features that segregate the two groups. The heatmap also visualizes co-occurring features, one example being the three SNV features lying in TACC3, which form their own cluster.

Additional file 1 contains the list of nonsynonymous exonic SNVs selected in this dataset as well as their respective model loadings. Limiting analysis to genes where selected SNVS are located, $19.49 \%$ of gene/sample pairs showed significant ASE - an enrichment compared to that of the null distribution $(p<0.001,2.64 \mathrm{X}$ greater than the mean of the null distribution).

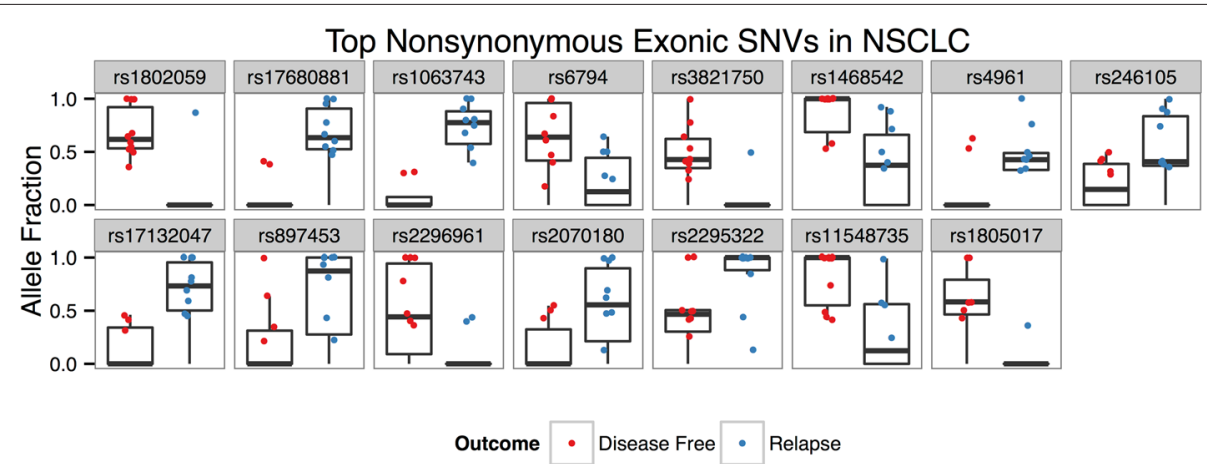

Fig. 2 NSCLC Exonic SNVS Alelle Fractions. Box plots of allele fraction distributions of the top 15 predictive SNVs identified during the creation of the nonsynonymous exonic SNV model in the NSCLC dataset. Only allele fractions $>0$ are plotted, though zero values contribute to box plot distributions 


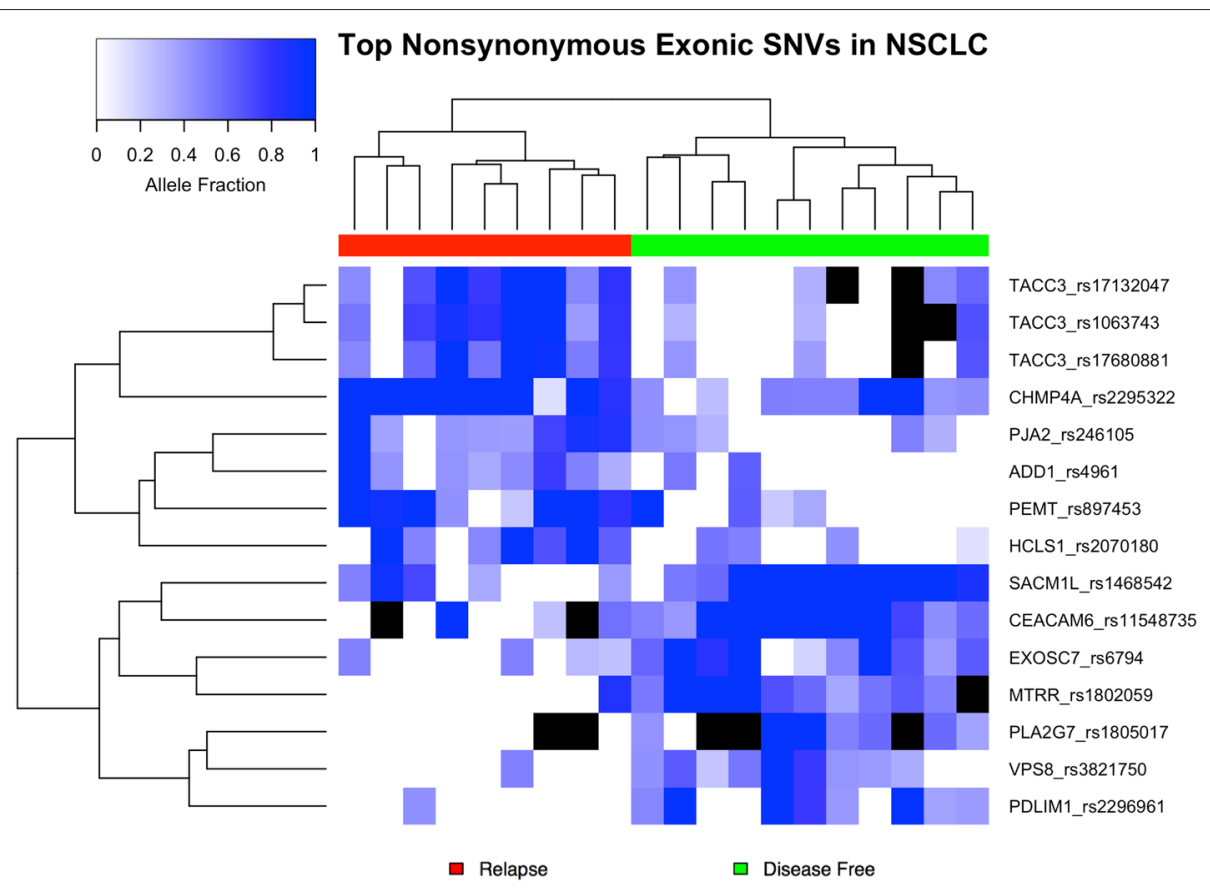

Fig. 3 Hierarchical Clustering of NSCLC Exonic SNVs A heatmap demonstrating the distinct clustering of sample groups and the co-occurrence of top nonsynonymous exonic SNV features in the NSCLC dataset. NA values are filled in with black

Table 5 contains annotations of the top 15 selected features. With 11 of the top 15 features being from genes that have previous associations with cancer, it is clear that the proposed methodology was able to identify features that have possible implications to cancer biology. Several examples include: CEACAM6, which is routinely used as a tumor marker in several cancers (including lung cancer) [9]; MTRR, in which variants have a well-documented association with increased risk of NSCLC [77]; and three SNVS in TACC3, whose high expression is associated with poor prognosis in NSCLC [37].

\section{Predictive SNV features: breast cancer hormone receptor status}

Except for up/downstream and 5'UTR models, the rankings of selected SNV features for each model were significantly different than univariate rankings from Fisher's exact test $\left(p s<10^{-6}\right)$. When comparing rankings to those produced by Wilcoxon rank sum test, all were significantly different $\left(p s<10^{-7}\right)$. The similarity to univariate rankings in the two models is likely a result of a small initial feature set size and/or the types of patterns seen in the data. For example, though the 5' UTR model produced rankings that were significantly different than rankings from Wilcoxon rank sum test, they were not significantly different than those from Fisher's exact test $(p=0.515)$, suggesting that predictive power of selected SNVs in this model result more from the differential abundance of AF values (number of nonzeros) than with the differential magnitude of AF values between groups, which Wilcoxon rank sum test seeks to quantify. The distribution of SNVs by region of origin selected during the training of the allSNVs model is given in Fig. 4. Notice that the majority of selected SNVs are located in traditional coding regions.

Additional files 2, 3, 4, 5, 6, 7 and 8 contain the lists of SNV features selected during the creation of each model. In the following sections, the top $15 \mathrm{SNVs}$ for selected models are highlighted to demonstrate that the genes in which they are reside are enriched for associations with cancer.

\section{Exonic}

Figure 5 visualizes allele fraction distributions of the top 15 predictive SNVs identified during the creation of the exonic SNV model in the breast cancer dataaset. SNVs chosen are more abundant in one of the groups and/or have higher AF values. Figure 6 demonstrates the clustering of samples by hormone receptor status. Though not a perfect clustering, the top 15 (of 50) features adequately segregate the two groups. Genes where selected exonic SNVs are located showed an enrichment of significant ASE events $-16.67 \%$ of gene/sample pairs $(p<0.001$, $1.75 \mathrm{X}$ greater than random mean).

Ten of the top 15 SNV features lie in genes that have previous associations with cancer; 6 , of which, are located in genes specifically associated with breast cancer (Table 6). Several outstanding examples include: HRPAP20, a hormone regulated breast cancer oncogene that promotes 
Table 5 Top 15 nonsynonymous exonic SNV features in NSCLC

\begin{tabular}{|c|c|c|c|c|c|}
\hline Gene & dbSNPID & Locus (Chr:bp) & Variant & Class & Description \\
\hline MTRR & rs1802059 & $5: 7878179$ & $\mathrm{C} \rightarrow \mathrm{T}$ & DF & Variants in gene are associated with NSCLC risk [77] \\
\hline TACC3 & rs17680881 & 4:1732978 & $\mathrm{G} \rightarrow \mathrm{A}$ & $\mathrm{R}$ & High expression of TACC3 associated with poor prognosis in NSCLC [37] \\
\hline TACC3 & rs1063743 & $4: 1729988$ & $\mathrm{G} \rightarrow \mathrm{A}$ & $\mathrm{R}$ & High expression of TACC3 associated with poor prognosis in NSCLC [37] \\
\hline EXOSC7 & rs6794 & 3:45052775 & $\mathrm{G} \rightarrow \mathrm{C}$ & DF & Downregulated in papillary thyroid cancer; component of exosome [31] \\
\hline VPS8 & rs3821750 & $3: 184766301$ & $\mathrm{~T} \rightarrow \mathrm{C}$ & DF & Vacular protein sorting 8 homolog [68] \\
\hline SACM1L & rs1468542 & 3:45779136 & $A \rightarrow T$ & DF & Phosphatidylinositol-4-phosphate phosphatase activity [68] \\
\hline ADD1 & rs4961 & 4:2906707 & $\mathrm{G} \rightarrow \mathrm{T}$ & $\mathrm{R}$ & $\begin{array}{l}\text { Specific variant is associated with hyper-tension [45]; regulates PPAR- } \gamma \\
\text { which is involved in cancer [44] }\end{array}$ \\
\hline PJA2 & rs246105 & 5:108672946 & $C \rightarrow T$ & $\mathrm{R}$ & $\begin{array}{l}\text { Presence of FER-PJA2 chimeras are associated with poor post-operative } \\
\text { NSCLC survival [41]; over-expressed in thryoid cancer [14] }\end{array}$ \\
\hline TACC3 & rs17132047 & 4:1729953 & $\mathrm{G} \rightarrow \mathrm{A}$ & $\mathrm{R}$ & Higher expression of TACC3 associated with poor prognosis in NSCLC [37] \\
\hline PEMT & rs897453 & $17: 17425631$ & $C \rightarrow T$ & $\mathrm{R}$ & Increased expression of PEMT in NSCLC patients predict shorter survival [89] \\
\hline CLIM1 & rs2296961 & 10:97023630 & $\mathrm{T} \rightarrow \mathrm{C}$ & DF & $\begin{array}{l}\text { Upregulated in breast cancer; cytoskeleton adapter protein; regulates estro- } \\
\text { gen receptor [36] }\end{array}$ \\
\hline HS1 & rs2070180 & 3:121351338 & $\mathrm{C} \rightarrow \mathrm{T}$ & $\mathrm{R}$ & Over-expression associated with poor survival in leukemia [12] \\
\hline CHMP4A & rs2295322 & $14: 24679877$ & $\mathrm{C} \rightarrow \mathrm{T}$ & $\mathrm{R}$ & Over-expression associated with recurrent ovarian cancer [7] \\
\hline CEACAM6 & rs11548735 & 19:42265889 & $\mathrm{G} \rightarrow \mathrm{T}$ & DF & $\begin{array}{l}\text { Carcinoembryonic antigen-related cell adhesion molecule, tumor marker in } \\
\text { cancer [9] }\end{array}$ \\
\hline PLA2G7 & rs1805017 & $6: 46684222$ & $C \rightarrow T$ & DF & $\begin{array}{l}\text { Associated with aggressive prostate cancer [76]; specific variant association } \\
\text { with protection from coronary heart disease [83] }\end{array}$ \\
\hline
\end{tabular}

malignant tumor growth [40]; ARHGEF16, which promotes migration and invasion of breast cancer cells [29]; FASN, whose upregulation is associated with HER2+ tumors and metastatic lesions [38] (confirmed in this dataset: FC $=3.6, p<10^{-5}$, DESeq2); and USP35, amplification of which is associated with significantly worse prognosis in breast cancer patients and is associated with ER- tumors [21]. The latter demonstrates a seemingly paradoxical result as the SNV in question is largely abundant in ER+ tumors. In fact, in this dataset USP35 is significantly upregulated in $\mathrm{ER}+$ tumors $(\mathrm{FC}=4.2, p<$ $10^{-5}$ ), perhaps providing evidence that conflict with the

Distribution of SNVs by Type in All SNVs Model

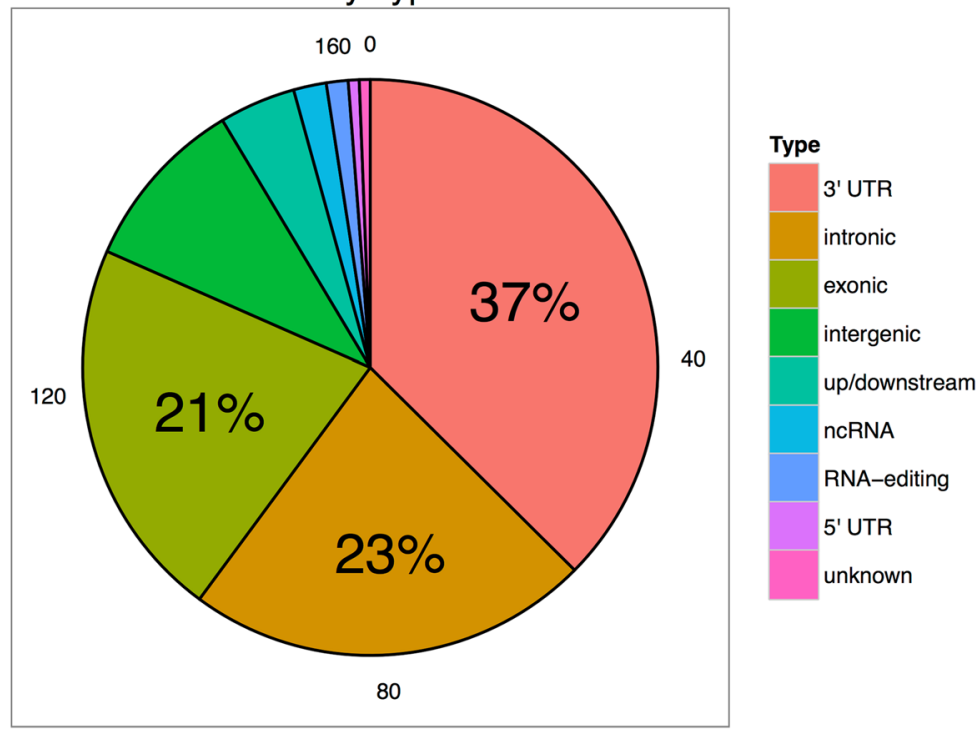

Fig. 4 Distribution of Selected SNV Features in All-SNVs Breast Cancer Model. 3'UTR, exonic, and intronic SNVs dominate the distribution of selected SNV features 


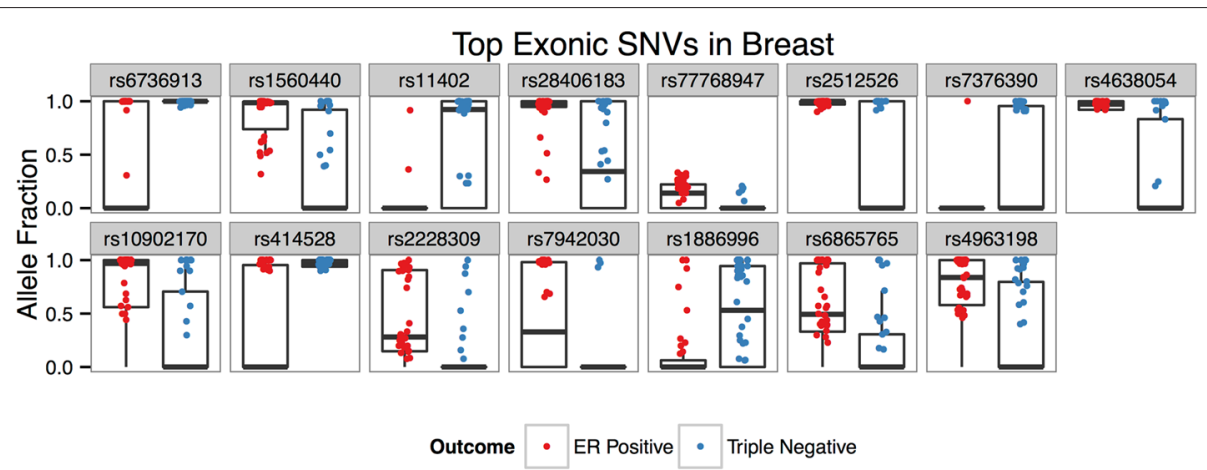

Fig. 5 Breast Exonic SNVs Alelle Fractions. Box plots of allele fraction distributions of top 15 predictive SNVs identified during the creation of the exonic SNV model in the breast cancer dataset. Only allele fractions $>0$ are plotted, though zero values contribute to box plot distributions

observation that USP35 amplification is associated with ER-tumors. It is important to note that the $5 \mathrm{SNV}$ features that do not have previous associations with cancer lie in genes that are uncharacterized, 3 of which from the same gene, LRRC56. These SNVs thus implicate genes that may provide future insight into the biology of the different cancer subtypes.

\section{Intronic}

Figure 7 visualizes allele fraction distributions of the top 15 predictive SNVs identified during the creation of the intronic SNV model in the breast cancer dataaset. The majority of identified SNV features have obvious differences in AF distributions, except for the SNV in HFM1 (chr1:91852851 A $\rightarrow$ G) which has small AF values. Interestingly, this gene is differentially expressed in this dataset with ER+ tumors expressing less reads $(\mathrm{FC}=-1.9, p=$ 0.011 ), suggesting a possible association of the SNV in the downregulation of the gene. Though not significant, the expression of HFM1 is decreased in samples in which the SNV is present (Student's t-test, $p=0.084$ ). Furthermore, selected intronic SNVs lie in genes that are enriched with ASE events, $24.04 \%$ of gene/sample pairs $(p<0.001$, $3.16 \mathrm{X}$ greater than random mean).

Eleven of the top $15 \mathrm{SNVs}$ are located in genes that have previous associations with cancer (Table 7); 9 of which are associated with breast cancer specifically. Some interesting examples include: $2 \mathrm{SNVs}$ that are located in

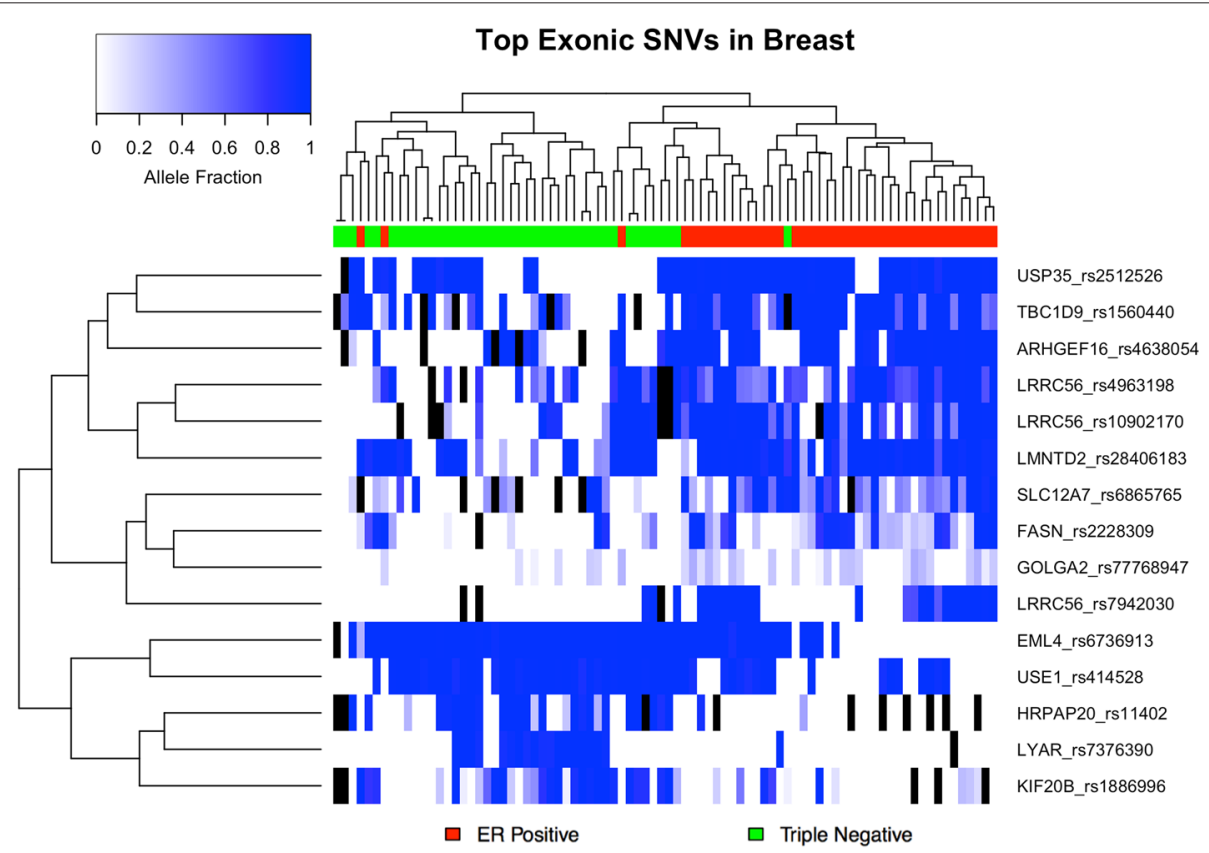

Fig. 6 Hierarchical Clustering of Breast Exonic SNVs. A heatmap demonstrating the distinct clustering of sample groups and the co-occurrence of top exonic SNV features in the breast cancer dataset. NA values are filled in with black 
Table 6 Top exonic SNVs in breast

\begin{tabular}{|c|c|c|c|c|c|c|}
\hline Gene & dbSNP ID & Locus (Chr:bp) & Variant & Type & Class & Description \\
\hline EML4 & rs6736913 & $2: 42510018$ & $\mathrm{~A} \rightarrow \mathrm{G}$ & NS & TR- & $\begin{array}{l}\text { EML4-ALK mutants are frequently found in NSCLC and some } \\
\text { breast cancers }[69,72]\end{array}$ \\
\hline TBC1D9 & rs1560440 & $4: 141543997$ & $\mathrm{G} \rightarrow \mathrm{A}$ & S & ER+ & A marker of tumor recurrence in breast cancer [3] \\
\hline HRPAP20 & rs11402 & 6:97339088 & $C \rightarrow T$ & S & TR- & $\begin{array}{l}\text { A hormone regulated oncogene in breast cancer that pro- } \\
\text { motes malignant tumor growth [40] }\end{array}$ \\
\hline LMNTD2 & rs28406183 & $11: 556521$ & $\mathrm{C} \rightarrow \mathrm{G}$ & S & ER+ & Uncharacterized lamin tail domain containing protein 2[68] \\
\hline GOLGA2 & rs77768947 & 9:131019765 & $C \rightarrow A$ & NS & ER+ & $\begin{array}{l}\text { Downregulation of GOLGA2/GM130 decreased angiogenesis } \\
\text { and cancer cell invasion in vitro and suppressed tumorigenesis } \\
\text { in lung cancer mice model [18] }\end{array}$ \\
\hline USP35 & rs2512526 & 11:77921527 & $\mathrm{G} \rightarrow \mathrm{C}$ & NS & ER+ & $\begin{array}{l}\text { Amplification of which is associated with significantly worse } \\
\text { prognosis in breast cancer and with ER- breast tumors [21] }\end{array}$ \\
\hline LYAR & rs7376390 & 4:4276132 & $\mathrm{T} \rightarrow \mathrm{C}$ & NS & TR- & Promotes invasion in colorectal cancer cells [81] \\
\hline ARHGEF16 & rs4638054 & $1: 3394456$ & $\mathrm{~T} \rightarrow \mathrm{C}$ & S & ER+ & Promotes migration and invasion of breast cancer cells [29] \\
\hline LRRC56 & rs10902170 & 11:554166 & $C \rightarrow G$ & NS & ER+ & Uncharacterized in humans [68] \\
\hline USE1 & rs414528 & 19:17330060 & $\mathrm{T} \rightarrow \mathrm{C}$ & NS & TR- & $\begin{array}{l}\text { A recently characterized SNARE protein, no characterized asso- } \\
\text { ciation with cancer phenotype [62] }\end{array}$ \\
\hline FASN & rs2228309 & 17:80051183 & $\mathrm{A} \rightarrow \mathrm{G}$ & S & ER+ & $\begin{array}{l}\text { Upregulation is associated with HER2+ tumors and metastatic } \\
\text { lesions [38] }\end{array}$ \\
\hline LRRC56 & rs7942030 & 11:549959 & $C \rightarrow T$ & S & ER+ & Uncharacterized in humans [68] \\
\hline KIF20B & rs1886996 & 10:91498127 & $T \rightarrow C$ & NS & TR- & Upregulation is associated with pancreatic cancer [4] \\
\hline SLC12A7 & rs6865765 & 5:1081702 & $\mathrm{A} \rightarrow \mathrm{G}$ & S & ER+ & $\begin{array}{l}\text { Also known as KCC4, in which LOF mutations significantly } \\
\text { inhibit xenograft tumors in SCID mice [30] }\end{array}$ \\
\hline LRRC56 & rs4963198 & $11: 551753$ & $\mathrm{G} \rightarrow \mathrm{A}$ & NS & ER+ & Uncharacterized in humans [68] \\
\hline
\end{tabular}

HDAC7, which has been shown to promote breast cancer cell survival and resistance to therapy [80]; 2 SNVs lying in CTBP1 and CTBP2, both of which are associated with breast cancer cell proliferation - the former being a regulator of BRCA [23, 53]; CD151, whose deregulation is predictive of poor outcome in node-negative lobular breast carcinoma [70]; and SNED1, high expression of which is correlated with poor outcome for ER-/PR- breast cancer patients [61] (interestingly SNED1 is significantly upregulated in $\mathrm{ER}+$ in this dataset: $\mathrm{FC}=2.8, p<10^{-4}$ ).
Also of note, several variants lie in genes that were previously implicated in the exonic model: two SNVs in ARHGEF16, one in EML4, and one in LMNTD2.

\section{3' UTR}

Figure 8 visualizes allele fraction distributions of the top 15 predictive SNVs identified during the creation of the 3' UTR SNV model in the breast cancer dataset demonstrating differential abundance and/or AF distributions. $20.41 \%$ of gene/sample pairs were enriched with

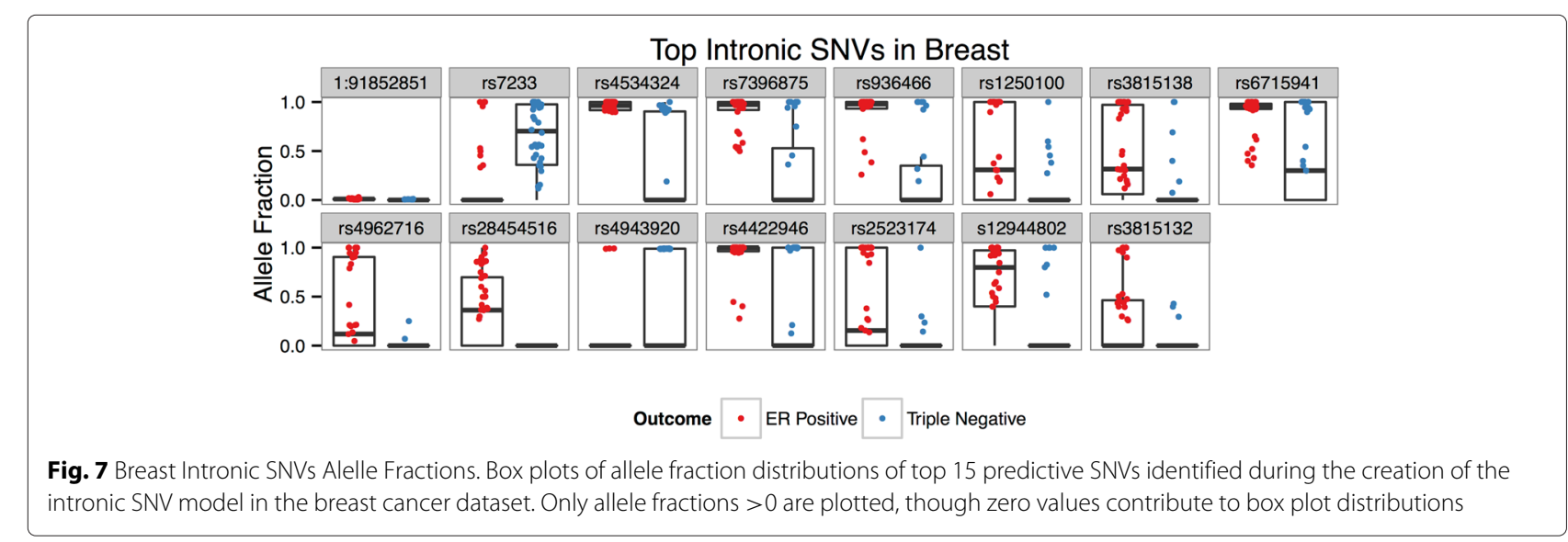


Table 7 Top intronic SNVs in breast

\begin{tabular}{|c|c|c|c|c|c|}
\hline Gene & dbSNPID & Locus (Chr:bp) & Variant & Class & Description \\
\hline HFM1 & NA & $1: 91852851$ & $A \rightarrow G$ & $\mathrm{ER}+$ & A DNA helicase that is specifically expressed in germline cells [75] \\
\hline EML4 & rs7233 & 2:42396722 & $A \rightarrow G$ & TR- & $\begin{array}{l}\text { EML4-ALK mutants are frequently found in NSCLC and some breast } \\
\text { cancers [69] }\end{array}$ \\
\hline ARHGEF16 & rs4534324 & 1:3394674 & $T \rightarrow C$ & $\mathrm{ER}+$ & Promotes migration and invasion of breast cancer cells [29] \\
\hline LRRC56 & rs7396875 & 11:551405 & $\mathrm{G} \rightarrow \mathrm{A}$ & ER+ & Uncharacterized in humans [68] \\
\hline LMNTD2 & rs936466 & 11:557342 & $\mathrm{T} \rightarrow \mathrm{C}$ & ER+ & Uncharacterized lamnin tail containing domain protein [68] \\
\hline CTBP1 & rs1250100 & 4:1236182 & $A \rightarrow G$ & ER+ & $\begin{array}{l}\text { Downregulates BRCA and E-cadherin in breast cancer, potential } \\
\text { biomarker for cancer development [23] }\end{array}$ \\
\hline HDAC7 & rs3815138 & 12:48178465 & $\mathrm{T} \rightarrow \mathrm{C}$ & ER+ & $\begin{array}{l}\text { Promotes breast cancer cell survival and therapy resistance by inhibiting } \\
\text { autophagic cell death [80] }\end{array}$ \\
\hline SNED1 & rs6715941 & 2:241993027 & $C \rightarrow T$ & ER+ & $\begin{array}{l}\text { High expression is correlated with poor outcome for ER-/PR- breast } \\
\text { cancer patients [61] }\end{array}$ \\
\hline CTBP2 & rs4962716 & 10:126685867 & $T \rightarrow C$ & ER+ & $\begin{array}{l}\text { High expression is associated with E-cadherin and cellular proliferation in } \\
\text { breast cancer [53] }\end{array}$ \\
\hline CD151 & rs28454516 & 11:833828 & $\mathrm{G} \rightarrow \mathrm{A}$ & ER+ & $\begin{array}{l}\text { Deregulation is predictive of poor outcome in node-negative lobular } \\
\text { breast carcinoma [70] }\end{array}$ \\
\hline DLG2 & rs4943920 & 11:85195154 & $A \rightarrow T$ & TR- & $\begin{array}{l}\text { The location of DLG2 is a common fragile site and is under expressed in } \\
\text { several cancers [57] }\end{array}$ \\
\hline ARHGEF16 & rs4422946 & 1:3394640 & $A \rightarrow G$ & $\mathrm{ER}+$ & Promotes migration and invasion of breast cancer cells [29] \\
\hline OAZ1 & rs2523174 & 19:2271181 & $T \rightarrow C$ & $\mathrm{ER}+$ & mRNA biomarker for oral cancer patients [20] \\
\hline GAA & rs12944802 & 17:78084418 & $\mathrm{G} \rightarrow \mathrm{A}$ & ER+ & $\begin{array}{l}\text { Acid alpha-glucosidase, which is essential for the degradation of glyco- } \\
\text { gen to glucose in lysosomes [68] }\end{array}$ \\
\hline HDAC7 & rs3815132 & $12: 48179048$ & $\mathrm{C} \rightarrow \mathrm{T}$ & ER+ & $\begin{array}{l}\text { Promotes breast cancer cell survival and therapy resistance by inhibiting } \\
\text { autophagic cell death [80] }\end{array}$ \\
\hline
\end{tabular}

significant ASE events $(p<0.001,1.71 \mathrm{X}$ greater than random mean).

Similar to the previously described models, the top 15 SNV features are located in genes enriched with cancer associations, 10 of 15 (Table 8); 6 of which are specifically associated with breast cancer. Some interesting examples include: NOTCH1, which has been shown to promote recurrence in breast cancer [1]; IKBKE, a breast cancer oncogene which is upregulated in TR- breast cancers [6]; LAMB1, a breast cancer biomarker [85]; PDXK, which is associated with breast cancer relapse and metastasis [35]; and COL1A1, which is upregulated in progesterone receptor positive breast cancer patients [50]. Interestingly, the top predictive SNV, rs11515 (a SNP located in the tumor suppressor gene CDKN2A), is associated with poor survival in glibolastoma patients and is moderately associated with breast cancer risk [24, 71]. Also of note, one of the SNVs lies in LRRC56, which has appeared in the top selected features in the two previous models.

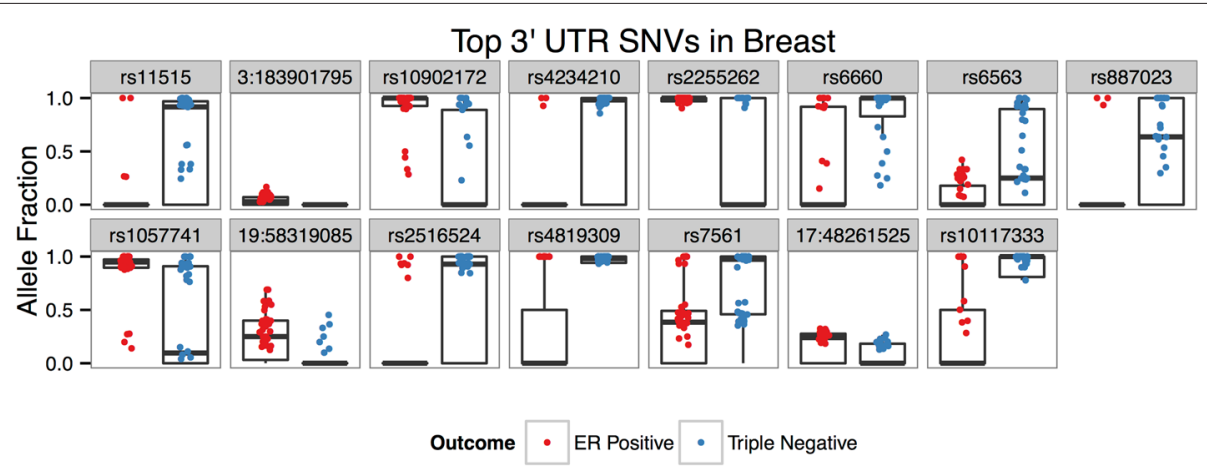

Fig. 8 Breast 3'UTR SNVS Alelle Fractions. Box plots of allele fraction distributions of top 15 predictive SNVs identified during the creation of the 3'UTR SNV model in the breast cancer dataset. Only allele fractions $>0$ are plotted, though zero values contribute to box plot distributions 
Table 8 Top 3' UTR SNVs in breast

\begin{tabular}{|c|c|c|c|c|c|}
\hline Gene & $\mathrm{dbSNPID}$ & Locus (Chr:bp) & Variant & Class & Description \\
\hline CDKN2A & rs11515 & 9:21968199 & $C \rightarrow G$ & TR- & $\begin{array}{l}\text { Moderate association with breast cancer risk [24]; rs11515 is associated } \\
\text { with poor survival in patients with glioblastoma multiforme [71] }\end{array}$ \\
\hline AP2M1 & NA & $3: 183901795$ & $\mathrm{~T} \rightarrow \mathrm{C}$ & ER+ & Over-expressed in espohageal squamous cell carcinoma [22] \\
\hline LRRC56 & rs10902172 & 11:554299 & $C \rightarrow G$ & ER+ & Uncharacterized in humans [68] \\
\hline SEC22A & rs4234210 & 3:122990805 & $\mathrm{G} \rightarrow \mathrm{A}$ & TR- & Vesicle trafficking protein [68] \\
\hline MTG1 & rs2255262 & 10:135234078 & $A \rightarrow G$ & $\mathrm{ER}+$ & $\begin{array}{l}\text { GTPase associated with ERBB4, which is associated with advanced NSCLC } \\
\text { [55] }\end{array}$ \\
\hline BTBD3 & rs6660 & 20:11907058 & $C \rightarrow T$ & TR- & Targeted by hsa-let-7i during colorectal cancer metastasis [87] \\
\hline $\mathrm{NOTCH} 1$ & rs6563 & 9:139389184 & $A \rightarrow G$ & TR- & Promotes recurrence in breast cancer [1] \\
\hline ZNF74 & rs887023 & 22:20761899 & $\mathrm{C} \rightarrow \mathrm{T}$ & TR- & Zinc finger protein [68] \\
\hline IKBKE & rs1057741 & $8: 42188550$ & $A \rightarrow G$ & ER+ & $\begin{array}{l}\text { Breast cancer oncogene [10]; upregulated in triple negative breast can- } \\
\text { cers [6] }\end{array}$ \\
\hline ZNF552 & NA & 19:58319085 & $\mathrm{T} \rightarrow \mathrm{C}$ & ER+ & Zinc finger protein [68] \\
\hline TRAPPC 10 & rs 2516524 & $21: 45525899$ & $A \rightarrow G$ & TR- & Transmembrane protein [68] \\
\hline PDXK & rs4819309 & $21: 45178438$ & $\mathrm{C} \rightarrow \mathrm{A}$ & TR- & Associated with breast cancer relapse and metastasis [35] \\
\hline LAMB1 & rs7561 & 7:107564366 & $\mathrm{T} \rightarrow \mathrm{G}$ & TR- & Identified as a secretome biomarker of breast cancer [85] \\
\hline COL1A1 & NA & 17:48261525 & $A \rightarrow T$ & ER+ & Upregulated in progesterone receptor positive patients [50] \\
\hline QSOX2 & rs10117333 & 9:139099451 & $A \rightarrow C$ & TR- & Upregulated in glioblastoma patients [5] \\
\hline
\end{tabular}

\section{RNA-editing}

Figure 9 visualizes allele fraction distributions of the 12 predictive SNVs identified during the creation of the model using SNVs located at known RNA-editing sites. Nine of the SNVs lie in genes that have previous associations with cancer (Table 9). The top SNV, a synonymous mutation lying in NEIL1, is the only exonic SNV chosen in the model. In fact, there is a paucity of exonic
SNVs in the total set $(n=8)$. Interestingly, RNA-editing sites and SNPs in this gene have been identified in other cancers [65]. Another interesting example is a SNV lying in NEAT1, a lncRNA whose overexpression is associated with poor prognosis in squamous cell carcinoma patients [19]. Intriguingly, one of the RNA-editing SNVs lies in ZNF552, which is also implicated in the 3' UTR and gene expression models.

Table 9 Top RNA-editing SNVs in breast

\begin{tabular}{|c|c|c|c|c|c|}
\hline Gene & Locus (Chr:bp) & Variant & Type & Class & Description \\
\hline NEIL1 & $15: 75646087$ & $A \rightarrow G$ & exonic & $\mathrm{ER}+$ & $\begin{array}{l}\text { A DNA repair protein in which polymorphisms and RNA-editing } \\
\text { sites have been reported in several cancers [65] }\end{array}$ \\
\hline ZNF552 & 19:58321691 & $\mathrm{T} \rightarrow \mathrm{C}$ & intronic & $\mathrm{ER}+$ & Zinc finger protein [68] \\
\hline $\mathrm{ABHD} 2$ & chr15:89744476 & $\mathrm{G} \rightarrow \mathrm{T}$ & 3' UTR & $\mathrm{ER}+$ & Upregulation is associated with colorectal cancer [84] \\
\hline RAB11FIP3 & chr16:548088 & $A \rightarrow G$ & intronic & $\mathrm{ER}+$ & Rab11-binding protein that regulates breast cancer cell motility [34] \\
\hline SMIM11 & chr21:35761073 & $\mathrm{G} \rightarrow \mathrm{T}$ & 3' UTR & TR- & Uncharacterized small integral membrane protein [34] \\
\hline PSMB2 & chr1:36068370 & $\mathrm{T} \rightarrow \mathrm{C}$ & 3' UTR & TR- & $\begin{array}{l}\text { A SNP, rs6661896, within this gene is associated with chronic } \\
\text { myelogenous leukemia [11] }\end{array}$ \\
\hline TPM4 & chr19:161915020 & $A \rightarrow G$ & intronic & TR- & $\begin{array}{l}\text { Upregulated in primary breast tumors compared to metastatic } \\
\text { lesions [59] }\end{array}$ \\
\hline NOM1 & chr7:156762883 & $A \rightarrow G$ & $3^{\prime}$ UTR & TR- & $\begin{array}{l}\text { Nucleolar Protein with MIF4G domain, involved with protein trans- } \\
\text { lation [68] }\end{array}$ \\
\hline NEAT1 & chr11:65208856 & $A \rightarrow G$ & intergenic & TR- & $\begin{array}{l}\text { A IncRNA whose overexpression is associated with poor prognosis } \\
\text { in squamous cell carcinoma patients [19] }\end{array}$ \\
\hline SEPT2 & chr2:242266408 & $A \rightarrow G$ & intronic & TR- & Expression is associated with hepatocellular cancer growth [16] \\
\hline LANCL2\&VOPP1 & chr7:55525773 & $\mathrm{T} \rightarrow \mathrm{C}$ & intergenic & TR- & LANCL2 is a regulator of the oncogene AKT1[86] \\
\hline PLB2\&SDS & chr12:113828961 & $A \rightarrow G$ & intergenic & TR- & Uncharacterized locus \\
\hline
\end{tabular}




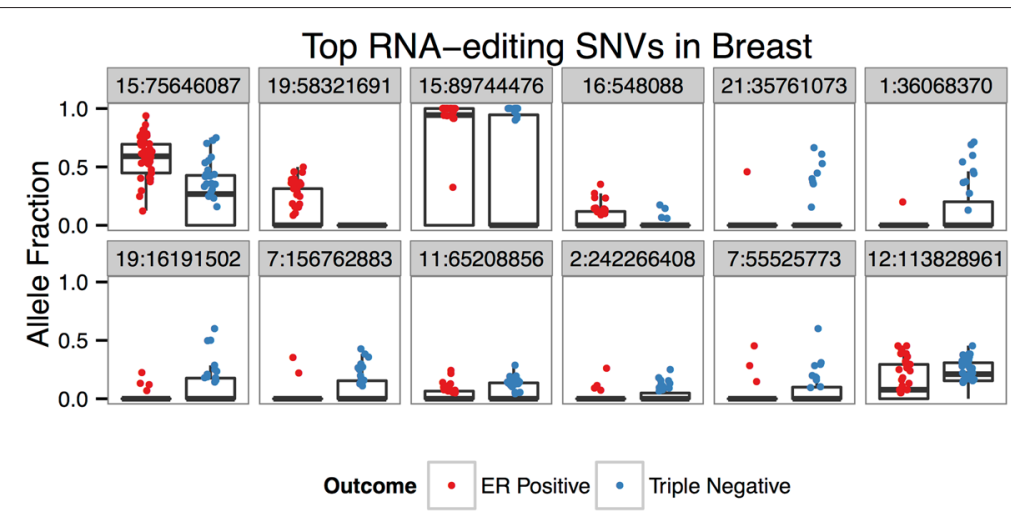

Fig. 9 Breast RNA-editing SNVs Alelle Fractions. Box plots of allele fraction distributions of 12 predictive SNVs identified during the creation of the RNA-editing SNV model in the breast cancer dataset. Only allele fractions $>0$ are plotted, though zero values contribute to box plot distributions

\section{Intergenic}

Something immediately noticeable about the features selected by this model is that 66 of the 771 features are located proximal to each other spanning 8,610 bp (chr9: 68 , 416, 905-68, 425, 515; hg19). Interestingly, 36 of these are further concentrated in a 1,635 bp region (chr9: 68, $418,108-68,419,742$ ) defined by a strong H3K27Ac peak and histone methylation peaks (H3K4me1, H3K4me3, H3K27me3, and H3K36me3) present in chronic myelogenous leukemia cell lines (K562), a moderate peak in human embryonic stem cells (H1-ESC), as well as a DNaseI hypersensitivity site and evidence of CTCF binding (Fig. 10) [25]. The enrichment of these peaks provide strong evidence that this locus is regulated in K562. Because this region is highly enriched with selected SNVs associated with the ER+ subtype, we have termed this locus estrogen receptor positive associated hotspot (ERPAHS). In fact, 9 of the top 15 selected intergenic SNVs are located in this concentrated region (Fig. 11). Furthermore, the only characterized transcripts within $100 \mathrm{kbp}$ of ERPAHS are two immediately flanking miRNAs (mir4477A and mir4477B) and a pseudogene
FRG1JP, all of unknown function [68], though the two miRNA were shown to be expressed by a subset of lymphoma cell lines in a published study [33].

To assay whether this region is regulated in ER+ and TR- breast samples, we sought to determine if the locus is differentially expressed. The location of ERPAHS (as defined by the 8,610 bp region $\pm 1,000$ bp; chr9: 68, $415,905-68,426,515)$ was included in the hg19 RefSeq annotation used by featureCounts during read assignment. Importantly, this region does not overlap the annotated transcripts mentioned above. Strikingly, this region is highly expressed in both ER+ and TR- breast samples and is upregulated in ER+ tumors $(\mathrm{FC}=1.63$, $p=0.0265$, Fig. 12). Furthermore, allele fraction values of the most predictive intergenic SNV, rs113539941 (chr9: 68418921 C $\rightarrow \mathrm{T}$ ), were significantly correlated with increased expression of this locus (Fig. 12). When comparing expression across samples classified with the binary presence of rs113539941, there is a more pronounced level of differential expression ( $\mathrm{FC}=2.67, p<10^{-5}$, Fig. 13), suggesting a functional role for associated mutations at this locus. This example provides a proof of concept of

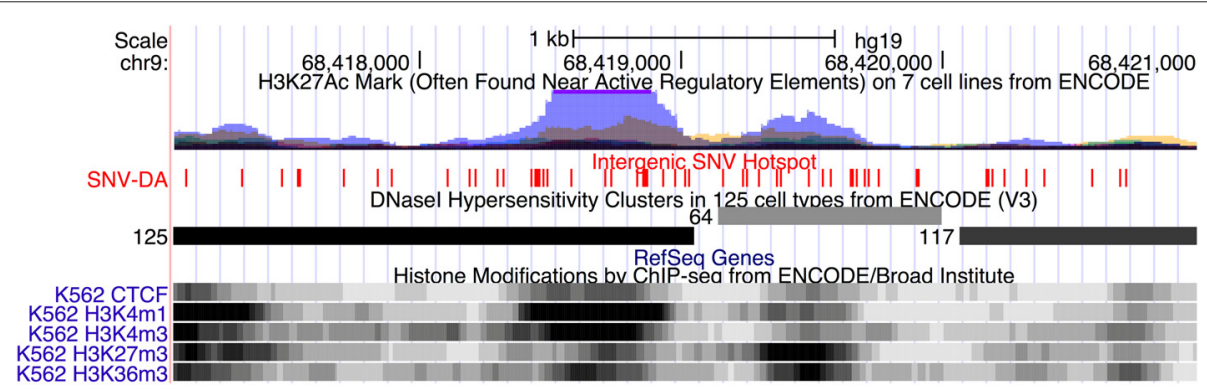

Fig. 10 Breast Intergenic Hotspot A UCSC genome browser view demonstrating the region of enriched selected intergenic SNVs in genomic position 9q12[43]. This locus is defined by the presence of several regulatory markers including CTCF binding, H3K27Ac, histone methylation marks, and a DNasel hypersensitvity site, all of which were found K562 cell lines. The enrichment of top selective intergenic SNV features suggests that this locus is associated and regulated in ER+ primary tumors 


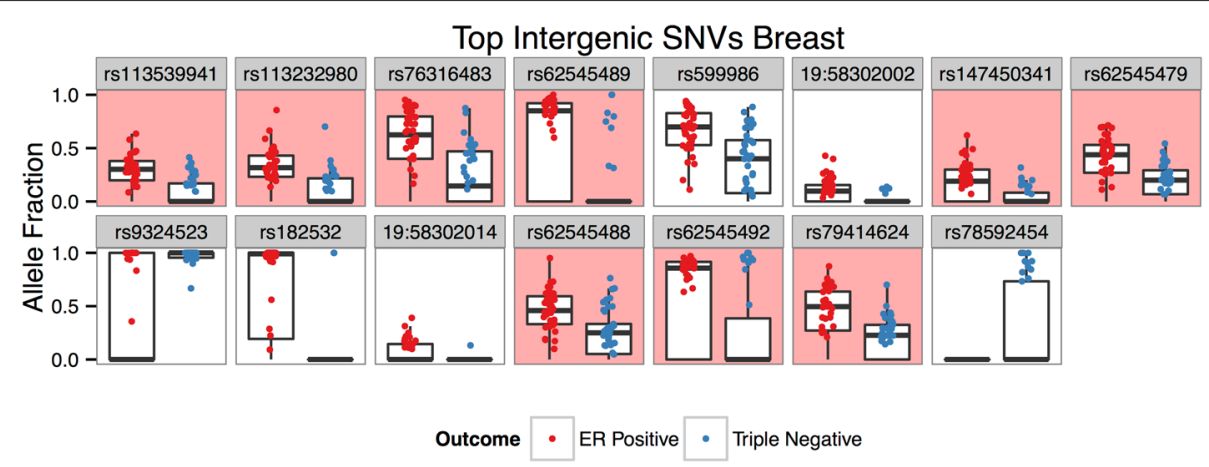

Fig. 11 Breast Intergenic SNVs Alelle Fractions. Box plots of allele fraction distributions of top 15 predictive SNVs identified during the creation of the intergenic SNV model in the breast cancer dataset. Only allele fractions $>0$ are plotted, though zero values contribute to box plot distributions. SNVs lying in the densely populated region of the estrogen positive associated hotspot are highlighted in red

the utility of the proposed approach to identify biologically associated regions, genes, and/or SNVs. In fact, there are 7 other regions identified via the selected intergenic SNVs that have at least 10 co-occurring SNVs. These loci provide potential new avenues for breast cancer research - further work should be done to identify their biological significance and functional roles in cancer.

\section{Conclusions}

This study introduces a new methodology and software pipeline, SNV-DA, which is used to identify differential patterns of mutation between two phenotypes from SNVs called from RNA-Seq data. In the breast cancer dataset, we demonstrated that SNV-DA was able to produce models with high predictive performances and that models were discriminant towards the true group labels, indicating that the methodology can identify and prioritize differentially abundant SNVs that are of biological interest. However, in the NSCLC dataset, the power of the proposed methodology to identify non-exonic SNVs that were predictive of disease outcome was likely limited by a combination of small sample size and/or shallow sequencing. We further demonstrated the utility of the proposed methodology by showing that selected SNV feature rankings were significantly different than univariate rankings by Fisher's exact and Wilcoxon rank sum tests and that the locations of selected SNVs were enriched with genes displaying significant allele-specific expression (Additional file 9). Though the relative performance of SNV models to traditional models trained on gene expression features varied, their performances were comparable - with the all-SNVs model producing the best classification accuracy of triple negative breast cancer samples (Additional file 10).

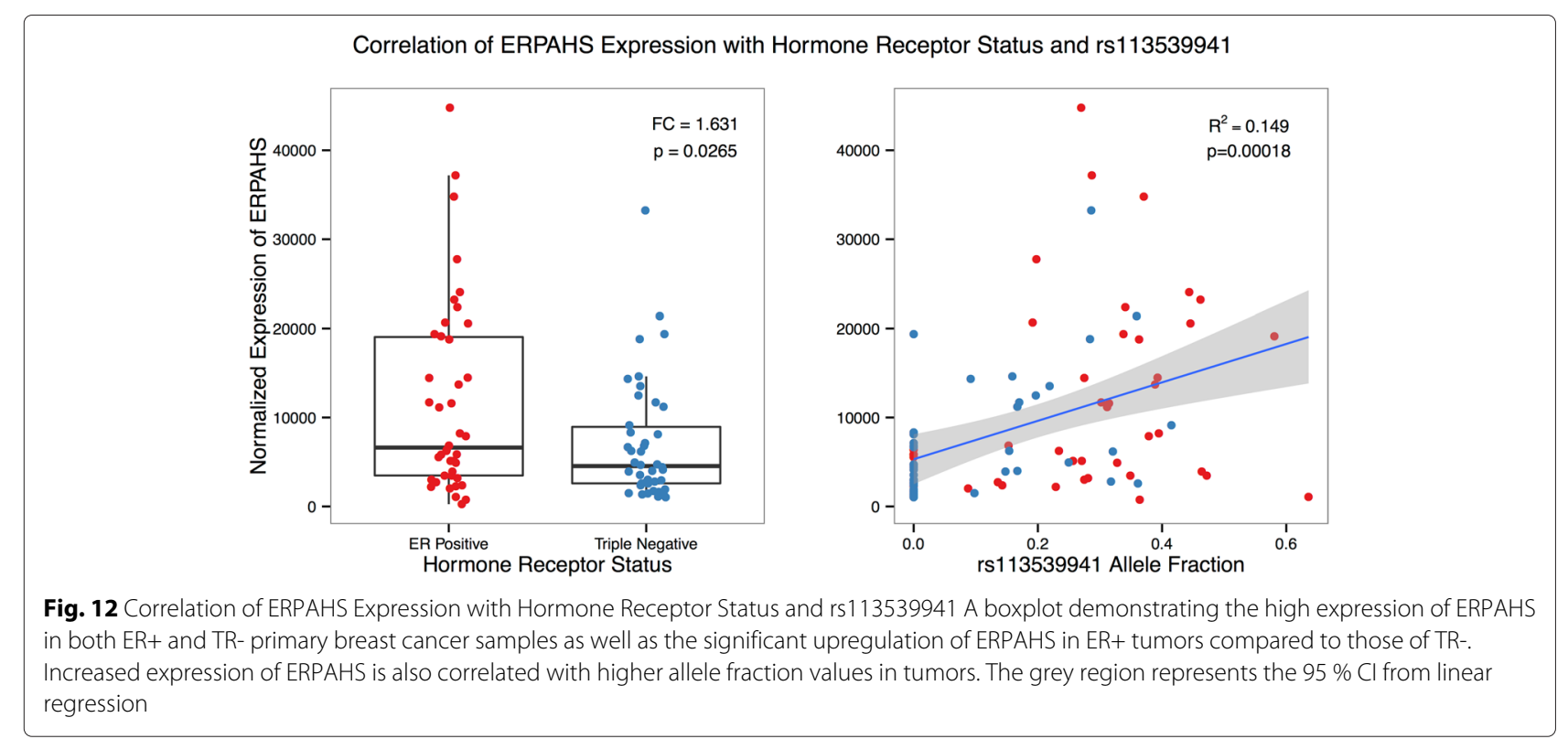




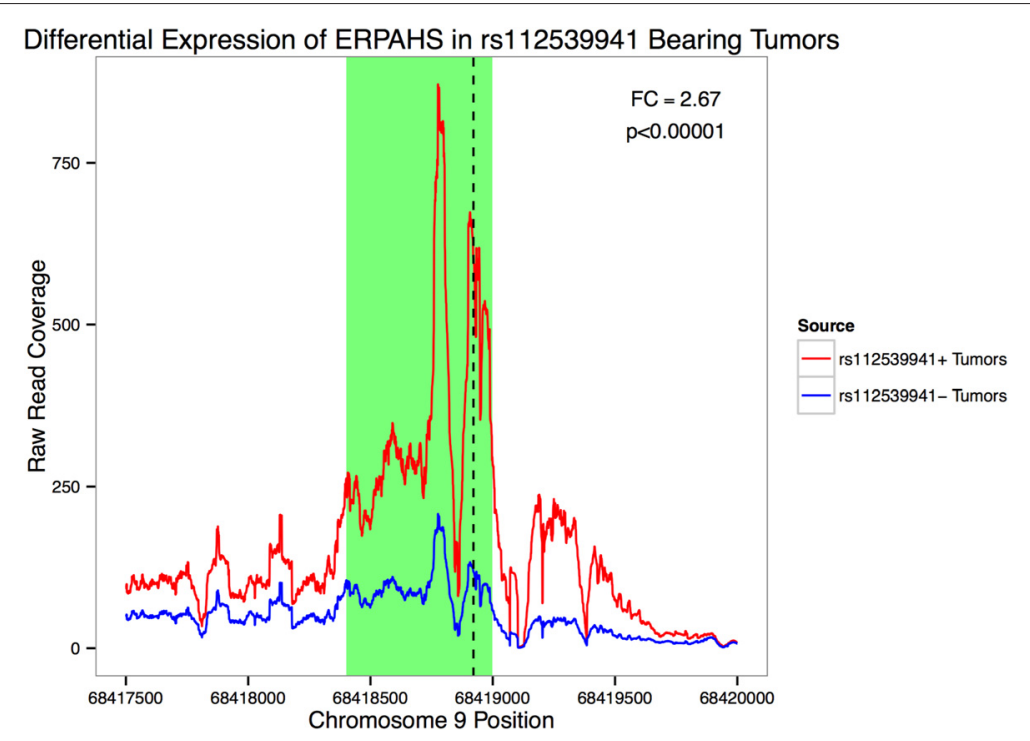

Fig. 13 Differential Expression of ERPAHS in Tumors Bearing rs113539941 Mean raw read coverage of tumors bearing rs1 13539941 and tumors lacking the SNV over the genomic location of ERPAHS. The approximate region of regulatory enrichment is highlighted in green with rs 113539941 marked with a dashed line. Fold change and significance of differential expression is given from DESeq2 using normalized read counts

Importantly, SNV-DA was able to identify small subsets of SNVs that can be used for further analysis (as little as 50 features in the breast exonic model). Characterization of top performing SNV features from optimally performing models demonstrated that there is an enrichment of SNVs originating from genes previously associated with cancer risk, progression, and survival. That the majority of selected features lie in genes that have strong relationships with the analyzed phenotype supports the use of SNV-DA for the identification and prioritization of novel molecular targets associated with disease phenotype. Furthermore, in the breast cancer dataset, SNV-DA was able to identify predictive SNVs located in ncRNA as well as intronic, 5' UTR, 3' UTR, up/downstream, and intergenic regions locations in the genome that are routinely ignored by whole exome sequencing. One outstanding example was the prioritization of the previously studied SNP, rs11515, located in the 3'UTR of the tumor suppressor CDKN2A, which has clear associations with poor prognosis and risk in different cancers [71]. SNV-DA was also able to implicate SNVs originating from RNA-editing, an analysis exclusive to RNA sequencing data. Lastly, the identification of the differentially expressed ERPAHS locus, its significant correlation with predictive SNV allele fractions, and association with regulatory regions in the K562 cancer cell line demonstrates the utility of the proposed methodology for the identification of interesting unannotated expressed regions of the genome (also ignored by traditional differential gene expression analyses).

Because RNA-Seq variant calling is limited to regions of the genome that are expressed, the proposed methodology would not be able to identify variants that result in a marked decrease of expression below a threshold level. However, SNVs called from RNA-Seq data have the added benefit over traditional whole-genome or exome sequencing in that they provide information on the relative amounts of allelic expression, which - as shown - can be implicated in disease or phenotype. Moreover, this methodology can also be used to analyze WGS and WES data to determine the differential abundance of alleles across heterogenous tissues. Furthermore, the developers of GATK have recently outlined best practices for indel calling from RNA-Seq data; therefore, SNV-DA can also be used for the analysis of those variants where special attention is given to feature definitions (over-lapping intervals, etc). Though SNV-DA identified predictive SNVs lying in miRNA and lncRNA, the SNPiR pipeline is not designed for non-standard RNA-Seq methodologies, such as small RNA-Seq. Consequently, the development of novel variant calling methods for these non-standard approaches may produce new avenues of research that are amenable to classification using SNV-DA. A tremendous amount of RNA-Seq data has already been collected in the private and public domain. This data now has the added benefit in that it can be mined for more clinical insights via SNV-DA.

\section{Availability of supporting data}

SNV-DA is freely available at https://github.com/ Anderson-Lab/SNV-DA. Data transformation is implemented in Python and parallelized model creation, evaluation, and permutation tests are implemented in $\mathrm{R}$. 
The package allows users to specify cross-validation design, filtering criteria, and values of $\mathrm{K}$ to be tested, as well as the automatic creation of supporting figures. The repository also contains variant data used in this study, as well as the necessary code and documentation to run SNV-DA and SNV calling from RNA-Seq data.

\section{Additional files}

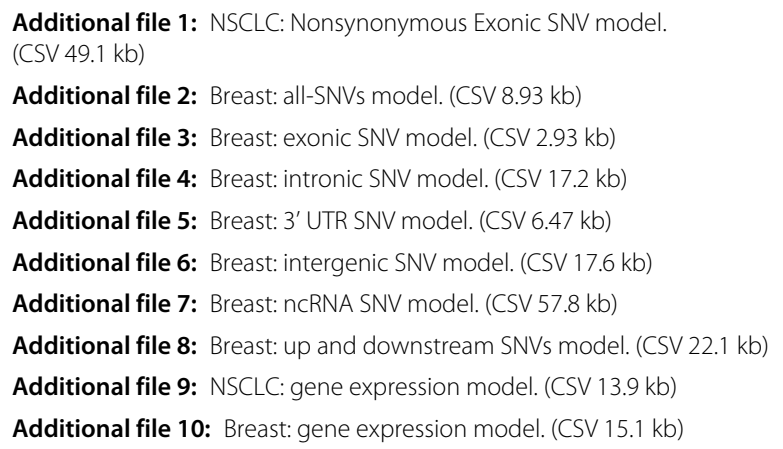

\section{Abbreviations}

AF: allele fraction; ASE: alelle-specific expression; AUC: area under the receiver operating characteristic curve; BWA: Burrows-Wheelers Aligner; DGE: differential gene expression; DF: Disease Free; ER+: estrogen receptor positive; ERPAHS: ER+ associated hotspot; GATK; genome analysis toolkit; NA: non-applicable; NSCLC: non-small cell lung cancer; R: relapse; RNA-Seq: RNA sequencing; SNV: single nucleotide variant; SNV-DA: SNV-discriminant analysis; SNVM: SNV matrix; SPLS-DA: sparse projections to latent structures discriminant analysis; TR-: triple-negative; WES: whole-exome sequencing; WGS: whole-genome sequencing.

\section{Competing interests}

The authors declare that they have no competing interests.

\section{Authors' contributions}

MP and PA were involved in the overall design and conception of the project, methodology development, statistical analysis of all data sets, and wrote the manuscript with the help of all other authors. MP wrote and designed the SNV-DA pipeline with contributions and guidance from PA, NL, and DM. DW, PW, and RW provided biological samples and sequencing data, as well discussion during analysis and SNV-DA development. CD provided the de-identified samples in the NSCLC dataset for this study. All authors have read and approved this manuscript.

\section{Acknowledgements}

We acknowledge support from the Genomics Shared Resource, Hollings Cancer Center, Medical University of South Carolina. This shared resource is supported in part by the Hollings Cancer Center, Medical University of South Carolina Support Grant (P30 CA 138313). We also acknowledge support from the Genomics and Computational Biology graduate group at the University of Pennsylvania as well as the lab of Dr. Lewis Chodosh.

\section{Author details \\ 'Department of Computer Science, College of Charleston, 66 George St., Charleston, SC, USA. ${ }^{2}$ Hollings Cancer Center, Medical University of South Carolina, 165 Canon St., Charleston, SC, USA. ${ }^{3}$ Department of Pathology, Medical University of South Carolina, 165 Canon St., Charleston, SC, USA. ${ }^{4}$ Department of Surgery, Medical University of South Carolina, 165 Canon St., Charleston, SC, USA. ${ }^{5}$ Department of Cancer Biology, University of Pennsylvania, 421 Curie Blvd, Philadelphia, PA, USA.}

Received: 1 September 2015 Accepted: 25 February 2016 Published online: 31 March 2016

\section{References}

1. Abravanel DL, Belka GK, Pan TC, Pant DK, Collins MA, Sterner CJ, Chodosh LA. Notch promotes recurrence of dormant tumor cells following HER2/neu-targeted therapy. J Clin Investig. 2015;125(6): 2484-96.

2. Anderson P, Paul MR, McCaffrey V, Hazard E, Watson PM, Wilson R, Denlinger $C E$, Watson DK. Predictive modeling of lung cancer recurrence using alternative splicing events versus differential expression data. Comput Intell Bioinforma Comput Biol IEEE Conf. 20141-8.

3. Andres SA, Brock GN, Wittliff JL. Interrogating differences in expression of targeted gene sets to predict breast cancer outcome. BMC Cancer. 2013;13:326.

4. Ansari D, Andersson R, Bauden MP, Andersson B, Connolly JB, Welinder C, Sasor A, Marko-Varga G. Protein deep sequencing applied to biobank samples from patients with pancreatic cancer. J Cancer Res Clin Oncol. 2015;141(2):369-80.

5. Autelitano F, Loyaux D, Roudières S, Déon C, Guette F, Fabre P, Ping Q, Wang $S$, Auvergne R, Badarinarayana V, Smith M, Guillemot JC, Goldman SA, Natesan S, Ferrara P, August P. Identification of Novel Tumor-Associated Cell Surface Sialoglycoproteins in Human Glioblastoma Tumors Using Quantitative Proteomics. PLoS ONE. 2014;9(10):e110316.

6. Barbie TH, Alexe G, Aref AR, Li S, Zhu Z, Zhang X, Imamura Y, Thai TC, Huang Y, Bowden M, Herndon J, Cohoon TJ, Fleming T, Tamayo P, Mesirov JP, Ogino S, Wong KK, Ellis MJ, Hahn WC, Barbie DA, Gillanders WE. Targeting an IKBKE cytokine network impairs triple-negative breast cancer growth. J Clin Investig. 2014;124(12):5411-23.

7. Barlin JN, Jelinic P, Olvera N, Bogomolniy F, Bisogna M, Dao F, Barakat RR, Chi DS, Levine DA. Validated gene targets associated with curatively treated advanced serous ovarian carcinoma. Gynecol Oncol. 2013;128(3): 512-7.

8. Basu S, Chen W, Tchou J, Mavi A, Cermik T, Czerniecki B, Schnall M, Alavi A. Comparison of Triple-negative and Estrogen Receptor positive/Progesterone Receptor-positive/HER2-negative Breast Carcinoma Using Quantitative Fluorine-18 Fluorodeoxyglucose/Positron Emission Tomography Imaging Parameters. 112. 2008;5:995-1000.

9. Blumenthal RD, Leon E, Hansen HJ, Goldenberg DM. Expression patterns of CEACAM5 and CEACAM6 in primary and metastatic cancers. BMC Cancer. 2007;7:2.

10. Boehm JS, Zhao JJ, Yao J, Kim SY, Firestein R, Dunn IF, Sjostrom SK, Garraway LA, Weremowicz S, Richardson AL, Greulich H, Stewart CJ, Mulvey LA, Shen RR, Ambrogio L, Hirozane-Kishikawa T, Hill DE, Vidal M, Meyerson M, Grenier JK, Hinkle G, Root DE, Roberts TM, Lander ES, Polyak K, Hahn WC. Integrative genomic approaches identify IKBKE as a breast cancer oncogene. Cell. 2007;129(6):1065-79.

11. Bruzzoni-Giovanelli H, González JR, Sigaux F, Villoutreix BO, Cayuela JM, Guilhot J, Preudhomme C, Guilhot F, Poyet JL, Rousselot P. Genetic polymorphisms associated with increased risk of developing chronic myelogenous leukemia. Oncotarget. 2015;6(34):36269-77.

12. Butrym A, Majewski M, Dzietczenia J, Kuliczkowski K, Mazur G. High expression of hematopoietic cell specific Lyn substrate-1 (HS1) predicts poor survival of B-cell chronic lymphocytic leukemia patients. Leuk Res. 2012;36(7):876-80.

13. Bylesjö M, Rantalainen M, Cloarec O, Nicholson JK, Holmes E, Trygg J. OPLS discriminant analysis: combining the strengths of PLS-DA and SIMCA classification. J Chemom. 2006;20(8-10):341-51.

14. Cantara S, D'Angeli F, Toti P, Lignitto L, Castagna MG, Capuano S, Prabhakar BS, Feliciello A, Pacini F. Expression of the ring ligase PRAJA2 in thyroid cancer. J Clin Endocrinol Metab. 2012;97(11):4253-9.

15. Cao KL, Boitard S, Besse P. Sparse PLS discriminant analysis: biologically relevant feature selection and graphical displays for multiclass problems. BMC Bioinforma. 2011;12(253).

16. Cao $L Q$, Shao $Z L$, Liang $H H$, Zhang DW, Yang XW, Jiang XF, Xue $P$. Activation of peroxisome proliferator-activated receptor- $\gamma$ (PPAR $\gamma$ ) inhibits hepatoma cell growth via downregulation of SEPT2 expression. Cancer Lett. 2015;359(1):127-35.

17. Chakravarti A. Single nucleotide polymorphisms: ... to a future of genetic medicine. Nature. 2001;409:822-3.

18. Chang SH, Hong SH, Jiang HL, Minai-Tehrani A, Yu KN, Lee JH, Kim JE, Shin JY, Kang B, Park S, Han K, Chae C, Cho MH. GOLGA2/GM130, cis-Golgi matrix protein, is a novel target of anticancer gene therapy. Mol Ther. 2012;20(11):2052-63. 
19. Chen X, Kong J, Ma Z, Gao S, Feng X. Up regulation of the long non-coding RNA NEAT1 promotes esophageal squamous cell carcinoma cell progression and correlates with poor prognosis. Am J Cancer Res. 2015;5(9):2808-15.

20. Cheng YS, Jordan L, Rees T, Chen HS, Oxford L, Brinkmann O, Wong D. Levels of potential oral cancer salivary mRNA biomarkers in oral cancer patients in remission and oral lichen planus patients. Clin Oral Investig. 2014;18(3):985-93.

21. Chin SF, Teschendorff AE, Marioni JC, Wang Y, Barbosa-Morais NL, Thorne NP, Costa JL, Pinder SE, van de Wiel MA, Green AR, Ellis IO, Porter PL, Tavaré S, Brenton JD, Ylstra B, Caldas C. High-resolution aCGH and expression profiling identifies a novel genomic subtype of ER negative breast cancer. Genome Biol. 2007;8(10):R215.

22. Dadkhah E, Naseh H, Farshchian M, Memar B, Sankian M, Bagheri R, Forghanifard MM, Montazer M, Kazemi Noughabi M, Hashemi M, Abbaszadegan MR. A cancer-array approach elucidates the immune escape mechanism and defects in the DNA repair system in esophageal squamous cell carcinoma. Arch Iran Med. 2013;16(8):463-70.

23. Deng Y, Deng H, Liu J, Han G, Malkoski S, Liu B, Zhao R, Wang XJ, Zhang Q. Transcriptional down-regulation of Brca1 and E-cadherin by CtBP1 in breast cancer. Mol Carcinog. 2012;51(6):500-5007.

24. Driver KE, Song H, Lesueur F, Ahmed S, Barbosa-Morais NL, Tyrer JP, Ponder BAJ, Easton DF, Pharoah, PDP and Dunning AM, for the Studies in Epidemiology and Risks of Cancer Heredity (SEARCH) Team. Association of single-nucleotide polymorphisms in the cell cycle genes with breast cancer in the British population. Carcinogenesis. 2008;29(2):333-41.

25. ENCODE Project Consortium. An integrated encyclopedia of DNA elements in the human genome. Nature. 2012;489(7414):57-74.

26. Furrukh M, Al-Moundhri M, Zahid KF, Kumar S, Burney I. Customised, individualised treatment of metastatic non-small-cell lung carcinoma. Sultan Qaboos Univ Med J. 2013;13:202-17.

27. George NI, Bowyer JF, Crabtree NM, Chang CW. An iterative leave-one-out approach to outlier detection in RNA-Seq data. PLoS ONE. 2015;10(6).

28. Haffty BG, Yang Q, Reiss M, Kearney T, Higgins SA, Weidhaas J, Harris L, Hait W, Toppmeyer D. Locoregional relapse and distant metastasis in conservatively managed triple negative early-stage breast cancer. J Clin Oncol. 2006;24:5652-7.

29. Hiramoto-Yamaki N, Takeuchi S, Ueda S, Harada K, Fujimoto S, Negishi $\mathrm{M}$, Katoh H. Ephexin4 and EphA2 mediate cell migration through a RhoG-dependent mechanism. J Cell Biol. 2010;190:461-77.

30. Hsu YM, Chou CY, Chen HH, Lee WY, Chen YF, Lin PW, Alper SL, Ellory JC, Shen MR. IGF-1 upregulates electroneutral K-Cl cotransporter KCC3 and KCC 4 which are differentially required for breast cancer cell proliferation and invasiveness. J Cell Physiol. 2007;210(3):626-36.

31. Igci YZ, Arslan A, Akarsu E, Erkilic S, Igci M, Oztuzcu S, Cengiz B, Gogebakan B, Cakmak EA, Demiryurek AT. Differential expression of a set of genes in follicular and classic variants of papillary thyroid carcinoma. Endocr Pathol. 2011;22(2):86-96.

32. Imielinski M, Berger AH, Hammerman PS, Hernandez B, Pugh TJ, Hodis E, Cho J, Suh J, Capelletti M, Sivachenko A, Sougnez C, Auclair D, Lawrence MS, Stojanov P, Cibulskis K, Choi K, de Waal L, Sharifnia T, Brooks A, Greulich H, Banerji S, Zander T, Seidel D, Leenders F, Ansén S, Ludwig C, Engel-Riedel W, Stoelben E, Wolf J, Goparju C, Thompson K, Winckler W, Kwiatkowski D, Johnson BE, Jänne PA, Miller VA, Pao W, Travis WD, Pass HI, Gabriel SB, Lander ES, Thomas RK, Garraway LA, Getz G, Meyerson M. Mapp Hallmarks Lung Adenocarcinoma Massively Parallel Sequencing/ Cell. 2012;150(6):1107-20.

33. Jima DD, Zhang J, Jacobs C, Richards KL, Dunphy $\mathrm{CH}$, Choi WWL, Au WY, Srivastava G, Czader MB, Rizzieri DA, Lagoo AS, Lugar PL, Mann KP, Flowers CR, Bernal-Mizrachi L, Naresh KN, Evens AM, Gordon LI, Luftig M, Friedman DR, Weinberg JR, Thompson MA, Gill Jl, Liu Q, How T, Grubor V, Gao Y, Patel A, Wu H, Zhu J, Blobe GC, Lipsky PE, Chadburn A, Dave SS. the Hematologic Malignancies Research Consortium (HMRC). Deep sequencing of the small RNA transcriptome of normal and malignant human $B$ cells identifies hundreds of novel microRNAs. Blood. 2010;116(23):e118-27.

34. Jing J, Tarbutton E, Wilson G, Prekeris R. Rab11-FIP3 is a Rab11-binding protein that regulates breast cancer cell motility by modulating the actin cytoskeleton. Eur J Cell Biol. 2009;88(6):325-41.

35. Johansson HJ, Sanchez BC, Forshed J, Stål O, Fohlin H, Lewensohn R, Hall P, Bergh J, Lehtiö J, Linderholm BK. Proteomics profiling identify
CAPS as a potential predictive marker of tamoxifen resistance in estrogen receptor positive breast cancer. Clin Proteomics. 2015;12(1):8.

36. Johnsen $S A$, Güngör $C$, Prenzel $T$, Riethdorf $S$, Riethdorf $L$,

Taniguchi-Ishigaki N, Rau T, Tursun B, Furlow JD, Sauter G, Scheffner M, Pantel K, Gannon F, Bach I. Regulation of estrogen-dependent transcription by the LIM Cofactors CLIM and RLIM in breast cancer. Cancer Res. 2009;69(1):128-36.

37. Jung CK, Jung JH, Park GS, Lee A, Kang CS, Lee KY. Expression of transforming acidic coiled-coil containing protein 3 is a novel independent prognostic marker in non-small cell lung cancer. Pathol Int. 2006;56(9):503-9.

38. Jung YY, Kim HM, Koo JS. Expression of lipid metabolism-related proteins in metastatic breast cancer. PLoS ONE. 2015;10(9):e0137204.

39. Kalari KR, Rossell D, Necela BM, Asmann YW, Nair A, Baheti S, Kachergus JM, Younkin CS, Baker T, Carr JM, Tang X, Walsh MP, Chai HS, Sun Z, Hart SN, Leontovich AA, Hossain A, Kocher JP, Perez EA, Reisman DN, Fields AP, Thompson EA. Deep sequence analysis of non-small cell lung cancer: integrated analysis of gene expression, alternative splicing, and single nucleotide variations in lung Adenocarcinomas with and without oncogenic KRAS mutations. Front Oncol. 2012;2:12.

40. Karpm CM, Shukla MN, Buckley DJ, Buckley AR. HRPAP20: a novel calmodulin-binding protein that increases breast cancer cell invasion. Oncogene. 2007;26(12):1780-8.

41. Kawakami M, Ishikawa R, Amano Y, Sunohara M, Watanabe K, Ohishi N, Yatomi Y, Nakajima J, Fukayama M, Nagase T, Takai D. Detection of novel paraja ring finger 2-fer tyrosine kinase mRNA chimeras is associated with poor postoperative prognosis in non-small cell lung cancer. Cancer Sci. 2013;104(11):1447-54.

42. Kent WJ. BLAT - the BLAST-like alignment tool. Genome Res. 2002;12(4): 656-64.

43. Kent WJ, Sugnet CW, Furey TS, Roskin KM, Pringle TH, Zahler AM, Haussler D. The human genome browser at UCSC. Genome Res. 2002;12(6):996-1006.

44. Kim JB, Wright HM, Wright M, Spiegelman BM. ADD1/SREBP1 activates PPARgamma through the production of endogenous ligand. Proc Natl Acad Sci USA. 1998:95(8):4333-7.

45. Kundu A, Anand A. Computational study of ADD1 gene polymorphism associated with hypertension. Cell Biochem Biophys. 2013;65(1):13-19.

46. Langmead B, Trapnell C, Pop M, Salzberg SL. Ultrafast and memory-efficient alignment of short DNA sequences to the human genome. Genome Biol. 2014;10(3):R25

47. Li B, Dewey CN. RSEM: accurate transcript quantification from RNA-Seq data with or without a reference genome. BMC Bioinforma. 2011;12:323.

48. Li H, Durbin R. Fast and accurate short read alignment with Burrows-Wheeler transform. Bioinformatics. 2009;25(14):1754-60.

49. Liao Y, Smyth GK, Shi W. featureCounts: an efficient general-purpose program for assigning sequence reads to genomic features. Bioinformatics. 2014;30(7):923-30.

50. Lin J, Goldstein L, Nesbit A, Chen MY. Influence of hormone receptor status on spinal metastatic lesions in breast cancer patients. World Neurosurg. 2016;85:42-48. [Epub ahead of print].

51. Listgarten J, Damaraju S, Poulin B, Cook L, Dufour J, Driga A, Mackey J, Wishart D, Greiner R, Zanke B. Predictive models for breast cancer susceptibility from multiple single nucleotide polymorphisms. Clin Cancer Res. 2004;10:2725-37.

52. Liu B, Liu Q, Song Y, Li X, Wang Y, Wan S, Zhang Z, Su H. Polymorphisms of HIF1A gene are associated with prognosis of early stage non-small-cell lung cancer patients after surgery. Med Oncol. 2014;31(4):877.

53. Liu X, Yao N, Qian J, Huang H. High expression and prognostic role of CAP1 and CtBP2 in breast carcinoma: associated with E-cadherin and cell proliferation. Med Oncol. 2014;31(3):878.

54. Love MI, Huber W, Anders S. Moderated estimation of fold change and dispersion for RNA-Seq data with DESeq2. Genome Biol. 2014;15(12).

55. Marquez-Medina D, Popat S. Afatinib: a second-generation EGF receptor and ErbB tyrosine kinase inhibitor for the treatment of advanced non-small-cell lung cancer. Futur Oncol. 2015;11(18):2525-40.

56. Mayba O, Gilbert HN, Liu J, Haverty PM, Jhunjhunwala S, Jiang Z, Watanabe C, Zhang Z. MBASED: allele-specific expression detection in cancer tissues and cell lines. Genome Biol. 2014;15(8):405.

57. McAvoy S, Ganapathiraju SC, Ducharme-Smith AL, Pritchett JR, Kosari F, Perez DS, Zhu Y, James CD, Smith DI. Non-random inactivation of large 
common fragile site genes in different cancers. Cytogenet Genome Res. 2007;118:260-9.

58. McKenna A, Hanna M, Banks E, Sivachenko A, Cibulskis K, Kernytsky A, Garimella K, Altshuler D, Gabriel S, Daly M, DePristo MA. The genome analysis toolkit: a MapReduce framework for analyzing next-generation DNA sequencing data. Genome Res. 2010;20:1297-1303.

59. Milioli HH, Santos Sousa K, Kaviski R, Dos Santos Oliveira NC, De Andrade Urban C, De Lima RS, Cavalli IJ, De Souza Fonseca Ribeiro EM. Comparative proteomics of primary breast carcinomas and lymph node metastases outlining markers of tumor invasion. Cancer Genomics Proteomics. 2015;12(2):89-101.

60. Misra SK, Wang X, Srivastava I, Imgruet MK, Graff RW, Ohoka A, Kampert TL, Gao H, Pan D. Combinatorial therapy for triple negative breast cancer using hyperstar polymer-based nanoparticles. Chem Commun (Cambridge). 2015;51(93):16710-16713. [Epub ahead of print].

61. Naba A, Clauser KR, Lamar JM, Carr SA, Hynes RO. Extracellular matrix signatures of human mammary carcinoma identify novel metastasis promoters. eLife. 2014;3:e01308.

62. Okumura AJ, Hatsuzawa K, Tamura T, Nagaya H, Saeki K, Okumura F, Nagao K, Nishikawa M, Yoshimura A, Wada I. Involvement of a novel Q-SNARE, D12, in quality control of the endomembrane system. J Biol Chem. 2006;281:4495-506.

63. Paz-Yaacov N, Bazak L, Buchumenski I, Porath HT, Danan-Gotthold M, Knisbacher BA, Eisenberg E, Levanon EY. Elevated RNA editing activity is a major contributor to transcriptomic diversity in tumors. Cell Rep. 2015;13(2):267-6

64. Piskol R, Ramaswami G, Li JB. Reliable identification of genomic variants from RNA-Seq data. Am J Hum Genet. 2013;93(4):641-51.

65. Prakash A, Carroll BL, Sweasy JB, Wallace SS, Doublié S. Genome and cancer single nucleotide polymorphisms of the Human NEIL1 DNA Glycosylase: activity, structure, and the effect of editing. DNA Repair (Amst). 2014;14:17-26.

66. Quinlan AR, Hall IM. BEDTools: a flexible suite of utilities for comparing genomic features. Bioinformatics. 2010;26(6):841-2.

67. Ramaswami G, Li JB. RADAR: a rigorously annotated database of A-to-I RNA editing. Nucleic Acids Res. 2014;42(D1):D109-D113.

68. Rebhan M, Chalifa-Caspi V, Prilusky J, Lancet D. GeneCards: integrating information about genes, proteins and diseases. Trends Genet. 1997;13(4):163.

69. Robertson FM, Petricoin lii EF, Van Laere SJ, Bertucci F, Chu K, Fernandez SV, Mu Z, Alpaugh K, Pei J, Circo R, Wulfkuhle J, Ye Z, Boley KM, Liu H, Moraes R, Zhang X, Demaria R, Barsky SH, Sun G, Cristofanilli M. Presence of anaplastic lymphoma kinase in inflammatory breast cancer. SpringerPlus. 2013;2:497.

70. Romanska HM, Potemski P, Krakowska M, Mieszkowska M, Chaudhri S, Kordek R, Kubiak R, Speirs V, Hanby AM, Sadej R, Berditchevski F. Lack of CD151/integrin $\alpha 3 \beta 1$ complex is predictive of poor outcome in node-negative lobular breast carcinoma: opposing roles of CD151 in invasive lobular and ductal breast cancers. Br J Cancer. 2015;113(9): 1350-1357. [Epub ahead of print].

71. Royds JA, Al Nadaf S, Wiles AK, Chen YJ, Ahn A, Shaw A, Bowie S, Lam F, Baguley BC, Braithwaite AW, MacFarlane MR, Hung NA, Slatter TL. The CDKN2A G500 allele is more frequent in GBM patients with no defined telomere maintenance mechanism tumors and is associated with poorer survival. PLoS ONE. 2011;6(10):e26737.

72. Shaw AT, Yeap BY, Mino-Kenudson M, Digumarthy SR, Costa DB, Heist RS, Solomon B, Stubbs H, Admane S, McDermott U, Settleman J, Kobayashi S, Mark EJ, Rodig SJ, Chirieac LR, Kwak EL, Lynch TJ, lafrate AJ. Clinical features and outcome of patients with non-small-cell lung cancer who harbor EML4-ALK. J Clin Oncol. 2009;27(26):4247-53.

73. Song JS, Kim YS, Kim DK, Park SI, Jang SJ. Global histone modification pattern associated with recurrence and disease-free survival in non-small cell lung cancer patients. Pathol Int. 2012;62:182-90.

74. Stewart BW, Wild CP. Word Cancer Report 2014. World Health Organ. 2014;5:1.

75. Tanaka K, Miyamoto N, Shouguchi-Miyata J, Ikeda JE. HFM1, the human homologue of yeast Mer3, encodes a putative DNA helicase expressed specifically in germ-line cells. DNA Seq. 2006;17(3):242-6.

76. Vainio $P$, Lehtinen $L$, Mirtti T, Hilvo M, Seppanen-Laakso T, Virtanen J, Sankila A, Nordling S, Lundin J, Rannikko A, Orešič M, Kallioniemi O, Iljin K. Phospholipase PLA2G7, associated with aggressive prostate cancer, promotes prostate cancer cell migration and invasion and is inhibited by statins. Oncotarget. 2013;5(3):742-50.

77. Van Dyke AL, Cote ML, Wenzlaff AS, Abrams J, Land S, lyer P, Schwartz AG. Chromosome 5p Region SNPs Are Associated with Risk of NSCLC among Women. J Cancer Epidemiol. 2009. 2009:Article ID 242151.

78. Varley KE, Gertz J, Roberts BS, Davis NS, Bowling KM, Kirby MK, Nesmith AS, Oliver PG, Grizzle WE, Forero A, Buchsbaum DJ, LoBuglio AF, Myers RM. Recurrent read-through fusion transcripts in breast cancer. Breast Cancer Res Treat. 2014;146:287-97.

79. Wang K, Li M, Hakonarson H. ANNOVAR: functional annotation of genetic variants from high-throughput sequencing data. Nucleic Acids Res. 2010;38:e164.

80. Wu MY, Fu J, Xiao X, Wu J, Wu RC. MiR-34a regulates therapy resistance by targeting HDAC1 and HDAC7 in breast cancer. Cancer Lett. 2014;354(2):311-9.

81. Wu Y, Liu M, Li Z, Wu XB, Wang Y, Wang Y, Nie M, Huang F, Ju J, Ma C, Tan R, Zen K, Zhang CY, Fu K, Chen YG, Wang MR, Zhao Q. LYAR promotes colorectal cancer cell mobility by activating galectin-1 expression. Oncotarget. 2015;6(32):32890-32901.

82. Xiong HY, Alipanahi B, Lee LJ, Bretschneider H, Merico D, Yuen RKC, Hua Y, Gueroussov S, Najafabadi HS, Hughes TR, Morris Q, Barash Y, Krainer AR, Jojic N, Scherer SW, Blencowe BJ, Frey BJ. The human splicing code reveals new insights into the genetic determinants of disease. Science. 2015;347(6218).

83. Xu L, Zhou J, Huang $S$, Huang $Y, L E Y$, Jiang $D$, Wang F, Yang $X, X u W$, Huang X, Dong C, Zhang L, Ye M, Lian J, Duan S. An association study between genetic polymorphisms related to lipoprotein-associated phospholipase A(2) and coronary heart disease. Exp Ther Med. 2013;5(3): $742-50$.

84. Yoshida T, Itoda M, Muto T, Miyaguchi K, Mogushi K, Shoji S, Shimokawa K, lida S, Uetake H, Ishikawa T, Sugihara K, Mizushima H, Tanaka H. Clinical omics analysis of colorectal cancer incorporating copy number aberrations and gene expression data. Cancer Informat. 2010;9:147-61.

85. Zawadzka AM, Schilling B, Cusack MP, Sahu AK, Drake P, Fisher SJ, Benz CC, Gibson BW. Phosphoprotein secretome of tumor cells as a source of candidates for breast cancer biomarkers in plasma. Mol Cell Proteomics. 2014;13(4):1034-49.

86. Zeng $\mathrm{M}$, van der Donk WA, Chen J. Lanthionine synthetase C-like protein 2 (LanCL2) is a novel regulator of Akt. Mol Biol Cell. 2014;25(24):3954-61.

87. Zhang P1, Ma Y, Wang F, Yang J, Liu Z, Peng J, Qin H. Comprehensive gene and microRNA expression profiling reveals the crucial role of hsa-let-7i and its target genes in colorectal cancer metastasis. Mol Biol Rep. 2012;39(2):1471-8.

88. Zienolddiny S, Skaug V. Single nucleotide polymorphisms as susceptibility, prognostic, and therapeutic markers of nonsmall cell lung cancer. Lung Cancer Targets Ther. 2011;2012(3):1-14.

89. Zinrajh D, Hörl G, Jürgens G, Marc J, Sok M, Cerne D. Increased phosphatidylethanolamine $\mathrm{N}$-methyltransferase gene expression in non-small-cell lung cancer tissue predicts shorter patient survival. Oncol Lett. 2014;7(6):2175-9.

\section{Submit your next manuscript to BioMed Central and we will help you at every step:}

- We accept pre-submission inquiries

- Our selector tool helps you to find the most relevant journal

- We provide round the clock customer support

- Convenient online submission

- Thorough peer review

- Inclusion in PubMed and all major indexing services

- Maximum visibility for your research

Submit your manuscript at www.biomedcentral.com/submit
Biomed Central 